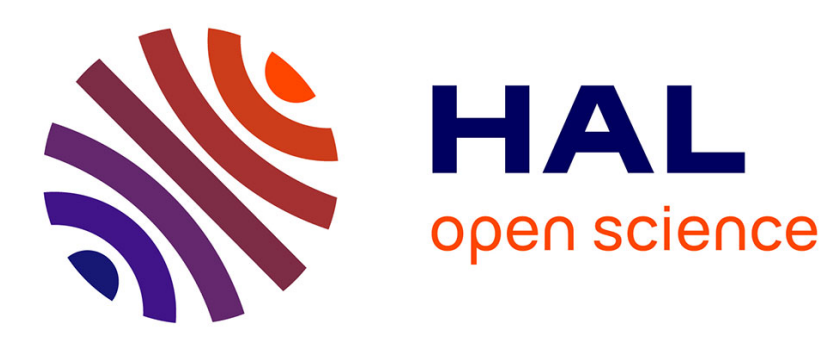

\title{
Multiparametric study of tearing modes in thin current sheets
}

H Betar, D del Sarto, M Ottaviani, A Ghizzo

\section{To cite this version:}

H Betar, D del Sarto, M Ottaviani, A Ghizzo. Multiparametric study of tearing modes in thin current sheets. Physics of Plasmas, 2020, 27, 10.1063/5.0022133 . hal-03320852

\section{HAL Id: hal-03320852 \\ https://hal.univ-lorraine.fr/hal-03320852}

Submitted on 16 Aug 2021

HAL is a multi-disciplinary open access archive for the deposit and dissemination of scientific research documents, whether they are published or not. The documents may come from teaching and research institutions in France or abroad, or from public or private research centers.
L'archive ouverte pluridisciplinaire HAL, est destinée au dépôt et à la diffusion de documents scientifiques de niveau recherche, publiés ou non, émanant des établissements d'enseignement et de recherche français ou étrangers, des laboratoires publics ou privés. 


\section{Multiparametric study of tearing modes in thin current sheets}

Cite as: Phys. Plasmas 27, 102106 (2020); https://doi.org/10.1063/5.0022133

Submitted: 17 July 2020 . Accepted: 10 September 2020 . Published Online: 14 October 2020

H. Betar, D. Del Sarto (D), M. Ottaviani, and A. Ghizzo (D)
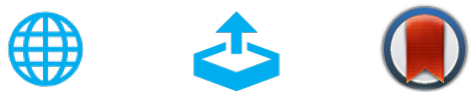

View Online

Export Citation

CrossMark

\section{ARTICLES YOU MAY BE INTERESTED IN}

Global ITG eigenmodes: From ballooning angle and radial shift to Reynolds stress and nonlinear saturation

Physics of Plasmas 27, 072507 (2020); https://doi.org/10.1063/5.0006765

Quasilinear modeling of heat flux from microtearing turbulence

Physics of Plasmas 27, 082306 (2020); https://doi.org/10.1063/5.0019082

Global gyrokinetic nonlinear simulations of kinetic infernal modes in reversed shear tokamaks

Physics of Plasmas 27, 092302 (2020); https://doi.org/10.1063/5.0013349

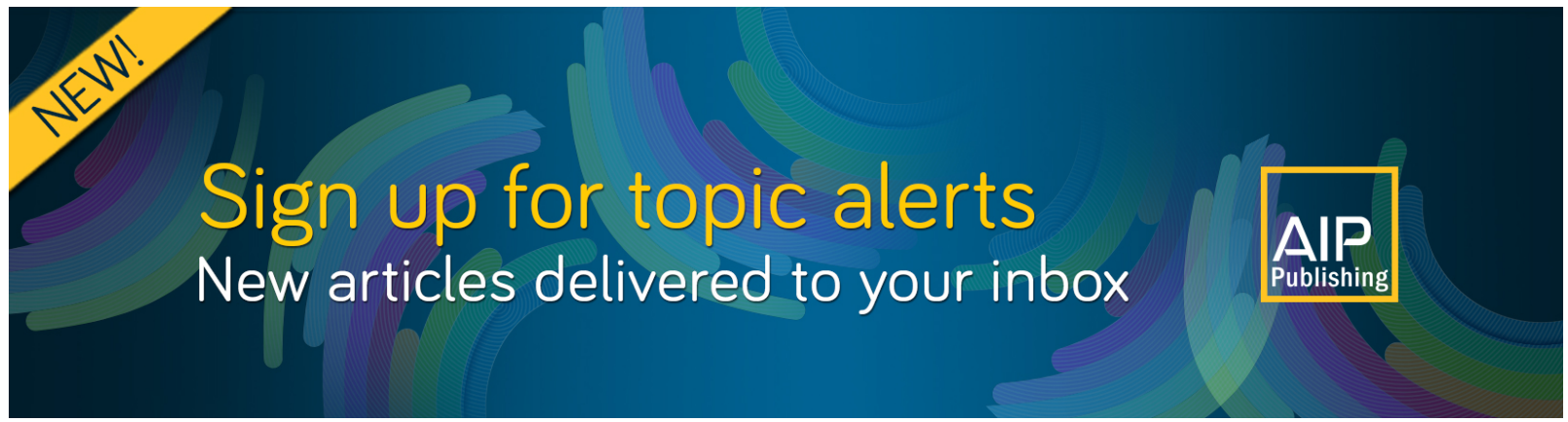




\title{
Multiparametric study of tearing modes in thin current sheets
}

\author{
Cite as: Phys. Plasmas 27, 102106 (2020); doi: 10.1063/5.0022133 \\ Submitted: 17 July 2020 - Accepted: 10 September 2020 . \\ Published Online: 14 October 2020

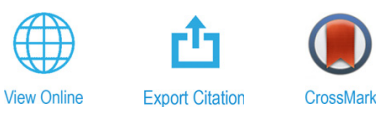 \\ H. Betar, ${ }^{1, a)}$ D. Del Sarto, ${ }^{7, b)}$ (D) M. Ottaviani, ${ }^{2, c)}$ and A. Ghizzo, ${ }^{1, d)}$ (D)
}

\author{
AFFILIATIONS \\ 'Institut Jean Lamour, UMR 7198 CNRS-Université de Lorraine, F-54000 Nancy, France \\ ${ }^{2}$ CEA, IRFM, F-13108 Saint-Paul-lez-Durance, France \\ a) Electronic mail: homam.betar@univ-lorraine.fr \\ b) Author to whom correspondence should be addressed: daniele.del-sarto@univ-lorraine.fr \\ ${ }^{c}$ Electronic mail: maurizio.ottaviani@cea.fr \\ ${ }^{d)}$ Electronic mail: alain.ghizzo@univ-lorraine.fr
}

\begin{abstract}
We investigate the asymptotic scaling of the growth rate and of the characteristic layer width of reduced-MHD tearing modes occurring in thin current sheets when reconnection depends on two non-ideal parameters. For this purpose, we use a new multi-precision finite difference eigensolver. The viscous-resistive regime, the warm-resistive regime that includes both resistivity and electron temperature effects, the warminertial regime in which a finite electron inertia replaces resistivity in allowing reconnection, and the inertial-resistive regime that includes both electron inertia and resistivity are investigated. Previous analytical results of the first three regimes are recovered. For all regimes, the scalings of the width of the reconnecting layer are provided in the different limits of the wavelength spectrum, and general estimates for the fastest growing modes are obtained and generalized to different magnetic equilibria. Implications for the disruption of evolving current sheets are discussed.
\end{abstract}

Published under license by AIP Publishing. https://doi.org/10.1063/5.0022133

\section{INTRODUCTION}

In this work, we carry out a numerical study of the stability of thin current sheets in a class of extended magnetohydrodynamic (MHD) models.

Thin current sheets can occur in laboratory plasmas, such as in the $m=1$ mode in tokamaks, and in a variety of space plasmas. They are also observed in numerical simulations of MHD turbulence. They are characterized by large aspect ratios $L / a$, with $L$ being the length of the sheet and $a$ its width, a background magnetic field $B_{0}$, and a sheet magnetic field $B_{c s}$, not necessarily of the same order as $B_{0}$.

Thin current sheets generally possess a broad range of unstable modes, ranging from a minimum wavenumber $k_{\min }$ of order $1 / L$ to a maximum wavenumber $k_{\max }$ of order $1 / a$. The spectrum has usually a maximum at a wavenumber $k_{M}$ such that $1 / L \ll k_{M} \ll 1 / a$. The maximum growth rate $\gamma_{M}$ is important to assess the eventual fate of the current sheet in the context of an evolving plasma. This notion will be discussed later in a more precise way. Also, in the following analysis, we will assume a continuum spectrum of wave-vectors $k$, which is formally valid only in the limit $k L \rightarrow \infty$.

The linear analysis is performed in the $2 \mathrm{D}$, slab geometry approximation of the reduced magnetohydrodynamic (MHD) model, which is valid in the strong guide field limit: we assume a sheared equilibrium magnetic field configuration $B_{y}(x)$ in the $x, y$ plane where we only consider reconnection modes that satisfy the condition $\partial / \partial z=0$, with $z$ being the direction of a uniform large guide field that ensures plasma incompressibility in the ideal case. With this restriction, for the configurations that we will investigate, reconnection can occur only at the resonant surface $x=0$, with $x$ being the inhomogeneity direction of the sheared magnetic fields.

We will investigate regimes where one non-ideal effect beside resistivity intervenes in the reconnection process: we will consider ion viscosity, electron temperature, and a finite electron inertia, respectively. Although considering just two of the non-ideal effects at a time may not represent more realistic regimes where all of them may play together, the present investigation can shed light on how these effects combine in allowing the onset and the development of reconnection. For this purpose, we have developed a multi-precision linear solver that uses finite difference schemes with tunable precision on a nonuniform grid with double periodic boundary conditions. It must also be noted that integration of the linear problem in regimes where the non-ideal parameters are sufficiently small, so as to justify the application of the asymptotic matching techniques of the boundary layer theory, is challenging also from a computational point of view because one must resolve strong spatial gradients in intervals of the domain 
with thickness that scales as some positive power of the small nonideal parameters.

This work is organized as follows:

Section II introduces the reduced-MHD (RMHD) equations we consider and the non-ideal parameters of the problem.

Section III discusses the linear limit of the equations and the numerical solver. Further details on the finite difference scheme and on the choice of the grid non-uniformity in relation to ill-conditioning of solutions and to machine precision are presented in Appendixes A and $B$, respectively. Details on the choice of the equilibrium profiles are given in Sec. III A.

Section IV recalls some general features about thin current sheet stability, which are reviewed in a qualitative manner for the purely resistive case.

Section V presents the results of the linear analysis in the viscousresistive regime, for which numerical results already available in the literature $^{1,2}$ have provided also a benchmark test for the solver.

Section VI is devoted to discuss the warm-resistive regime, where electron temperature effects combine with resistivity.

Section VII addresses the warm-inertial regime, with a focus on the comparison between electron inertia and resistivity in allowing reconnection.

Section VIII considers the inertial-resistive regime in which electron inertia combines with resistivity.

Section IX is devoted to the discussion of the results and their place in the understanding of the stability of thin sheets in evolving plasmas.

Section X summarizes the main points of the article and its motivation.

\section{MODEL EQUATIONS}

We consider the incompressible reduced-MHD model (hereafter, RMHD) where the dynamics of perturbations is dominated by Alfvén modes. We take its $2 \mathrm{D}$ limit, cold ion limit in slab geometry $(x, y)$. The model equations are as follows:

$$
\begin{gathered}
\frac{\partial F}{\partial t}+[\varphi, F]=\rho_{s}^{2}[U, \psi]+S^{-1} \nabla^{2} \psi, \\
\frac{\partial U}{\partial t}+[\varphi, U]=\left[\psi, \nabla^{2} \psi\right]+R^{-1} \nabla^{2} U .
\end{gathered}
$$

The previous system is non-dimensionalized using the reference magnetic field $\left(B_{0}\right)$, the reference length $a$ that represents the equilibrium magnetic field gradient scale, and the Alfvén time $\tau_{A}=a / v_{A}$, where the Alfvén velocity is given by $v_{A}=B_{0} / \sqrt{4 \pi m_{i} n_{0}}$. The "Poisson bracket" representation is given by $[f, g]=\partial_{x} f \partial_{y} g-\partial_{y} f \partial_{x} g$. The magnetic stream function $\psi$ is related (in dimensional units) to the magnetic field by $\boldsymbol{B}=\nabla \psi \times \boldsymbol{e}_{z}+B_{0} \boldsymbol{e}_{z}$, while the velocity stream function is related to the $\boldsymbol{E} \times \boldsymbol{B}$ velocity by $\boldsymbol{v}_{\boldsymbol{E} \times \boldsymbol{B}}=\nabla \varphi \times \boldsymbol{e}_{z}$. Also, $F=\psi-d_{e}^{2} \nabla^{2} \psi$ and $U=\nabla^{2} \varphi$. Here, $d_{e}=c /\left(\omega_{p e} a\right)$ is the electron skin depth normalized to $a$ (where $\omega_{p e}$ is the electron plasma frequency), $\rho_{s}=\left(\sqrt{m_{e} / m_{i}}\right) v_{t h}^{e} /\left(a \Omega_{i}\right)$ is the ion-sound Larmor radius normalized to $a$ (where $m_{\alpha}$ is the mass of the species, $\alpha=e, i, v_{t h}^{e}$ is the electron thermal velocity, and $\Omega_{i}$ is the ion cyclotron frequency), $R=a v_{A} / \nu$ is the Reynolds number related to ion-ion viscosity $\nu$, and $S=a v_{A} / \eta_{m}$ is the Lundquist number related to the magnetic diffusivity $\eta_{m}=c^{2} \eta /(4 \pi)$, where $\eta$ is the plasma resistivity. Note that the latter is in turn related to the electron-ion viscosity $\nu_{e i}$ : by $\eta=2 \pi \nu_{e i} / \omega_{p e}^{2}$ in dimensional units or by $S^{-1}=\nu_{e i} d_{e}^{2} / 2$ in dimensionless units.

Equations (1) and (2) have been derived in Refs. 3 and 4 in the collisional regime $\left(d_{e}=\rho_{s}=0\right)$ by relying on the assumption of a uniform, strong guide field along the $z$ direction. The parameter $\rho_{s}$, which violates the Lagrangian invariance of the parallel electron canonical momentum $F=\psi-d_{e}^{2} \nabla^{2} \psi$, is related to electron parallel compressibility effects. ${ }^{5}$ It is, however, also considered to be a FiniteLarmor-Radius (FLR) effect since in a strong guide field limit, it can be shown to be due to a non-isotropic component of the electron pressure tensor (see the early derivation of Ref. 6, in which it was obtained by including also a kinetic modeling of ion FLR effects, and a more recent fluid re-derivation in Ref. 7).

Note that the strong guide field hypothesis with which RMHD Eqs. (1) and (2) have been derived assumes fluctuations of the guide field to be ordered $\delta B_{z} \sim\left(\left|\boldsymbol{B}_{\perp}\right| / B_{0}\right)^{2}$, which are then negligible with respect to other quantities ordered $\sim\left|\boldsymbol{B}_{\perp}\right| / B_{0} \ll 1$. While the Hallterm contribution in Ohm's law is, therefore, completely neglected in the RMHD slab geometry here considered, it must be pointed out that alternative derivations of an identical set of equations exist, ${ }^{7-11}$ which relate the $\rho_{s}$-depending terms of (1) to the Hall-term in Ohm's law while assuming a standard polytropic (isothermal) closure for the electrons. In the latter kind of derivation, with respect to which [(1) and (2)] may be called "low- $\beta$ reduced Hall-MHD" equations, we must order $\delta B_{z} \sim\left|\boldsymbol{B}_{\perp}\right| / B_{0}$. In both cases, however, linearization of the system equations around a homogeneous equilibrium yields in the ideal MHD limit the dispersion relation of shear Alfvén waves, which become kinetic/inertial Alfvén waves (see, e.g., Ref. 12, pp. 20-21) when the microscopic scales $\rho_{s}$ and $d_{e}$ are attained and included. Further discussion on the role of plasma $\beta$ in RMHD orderings can be found in Ref. 70.

In conclusion, while throughout this article we will historically refer to the RMHD interpretation of Eqs. (1) and (2) with no Hall-term contribution, for which boundary layer calculations have been carried out in the past, the results we are going to discuss can be applied also to plasmas described by the low- $\beta$ reduced Hall-MHD model.

\section{LINEAR PROBLEM}

The system of equations (1) and (2) is linearized by introducing small perturbations of the form $f(x, y) \sim \tilde{f}(x) e^{i k y} e^{\gamma t}$ on static equilibrium profiles with $\psi_{0}=\psi_{0}(x) \neq 0$ and $\varphi_{0}=0$. This leads to the following system:

$$
\begin{aligned}
\gamma\left[1-d_{e}^{2}\left(\frac{\partial^{2}}{\partial x^{2}}-k^{2}\right)\right] \psi_{1} & =-i k \rho_{s}^{2} \psi_{0}^{\prime}\left(\frac{\partial^{2}}{\partial x^{2}}-k^{2}\right) \varphi_{1} \\
& +i k F_{0}^{\prime} \varphi_{1}+S^{-1}\left(\frac{\partial^{2}}{\partial x^{2}}-k^{2}\right) \psi_{1} \\
\gamma\left(\frac{\partial^{2}}{\partial x^{2}}-k^{2}\right) \varphi_{1}= & i k\left[\psi_{0}^{\prime}\left(\frac{\partial^{2}}{\partial x^{2}}-k^{2}\right)-\psi_{0}^{\prime \prime \prime}\right] \psi_{1} \\
+ & {\left[R^{-1}\left(\frac{\partial^{4}}{\partial x^{4}}-2 k^{2} \frac{\partial^{2}}{\partial x^{2}}+k^{4}\right)\right] \varphi_{1} . }
\end{aligned}
$$

Different reconnection regimes can be studied this way. In the following, we will focus on collisional regimes in which only two parameters at a time will be chosen to be different from zero: $S$ and $R$, 
$S$ and $\rho_{s}$, and $S$ and $d_{e}$. Equations (3) and (4) can be written in the following form:

$$
\begin{gathered}
\gamma\left(\begin{array}{cc}
\mathscr{D} & 0 \\
0 & i \mathscr{A}
\end{array}\right)\left(\begin{array}{c}
\psi \\
\varphi
\end{array}\right)=\left(\begin{array}{cc}
\mathscr{L}_{1} & \mathscr{L}_{2} \\
\mathscr{L}_{3} & \mathscr{L}_{4}
\end{array}\right)\left(\begin{array}{l}
\psi \\
\varphi
\end{array}\right), \\
\mathscr{A}=\frac{\partial^{2}}{\partial x^{2}}-k^{2}, \quad \mathscr{D}=1+k^{2} d_{e}^{2}-d_{e}^{2} \frac{\partial^{2}}{\partial x^{2}}, \quad \mathscr{L}_{1}=S^{-1} \mathscr{A}, \\
\mathscr{L}_{2}=k \rho_{s}^{2} \psi_{0}^{\prime} \mathscr{A}-k F_{0}^{\prime}, \quad \mathscr{L}_{3}=k \psi_{0}^{\prime} \mathscr{A}-i k \psi_{0}^{\prime \prime \prime}, \\
\mathscr{L}_{4}=i R^{-1} \mathscr{E}, \text { and } \mathscr{E}=\frac{\partial^{4}}{\partial x^{4}}-2 k^{2} \frac{\partial^{2}}{\partial x^{2}}+k^{4} .
\end{gathered}
$$

An exact analytical solution to this general eigenvalue problem does not generally exist in a closed form, but approximated analytical solutions, using a boundary layer approach, can be obtained in the purely resistive constant- $\psi$ regime ${ }^{13}$ and internal kink ${ }^{14,15}$ regimes or in purely collisionless reconnection regimes. ${ }^{16-18}$ Heuristic estimations (cf. Sec. IV) can be sometimes used to evaluate the scalings of the growth rates and of the reconnecting layer width by relying on an appropriate balancing of the terms of Eqs. (3) and (4), without working out all the calculations of the asymptotic matching of the boundary layer approach. This kind of simplified analysis has been first applied to kinetic tearing equations. ${ }^{19}$ Even in this case, however, the support of numerical calculations may be required, as for regimes in which finite Larmor radius effects are included (this point will be addressed in detail in a forthcoming article).

A numerical approach to the eigenvalue problem (5) is, therefore, useful to determine the scalings and eigenmode profiles in different reconnection regimes. For this, one needs to resolve accurately the thin layer (hereafter named the "inner layer") around the reconnecting current sheet, where non-ideal MHD effects become important. We indicate with $\delta$ the thickness of such a layer.

To this purpose, we have developed an eigensolver for a slab periodic box of dimensions $\left[-L_{x} / 2, L_{x} / 2\right] \times\left[-L_{y} / 2, L_{y} / 2\right]$, which uses compact finite difference schemes of tunable precision for the derivatives (see Appendix A) on a non-uniform grid along the $x$-direction (see Appendix B). The non-uniformity of the grid ensures the spatial resolution of the inner layer, where the grid spacing can be chosen to be much smaller than in the outer layer, where the non-ideal parameters can be neglected. While this guarantees a faster convergence of the solution of the eigenvalue problem, it also leads to ill-conditioned finite difference matrices when the non-uniformity is high and the non-ideal parameters take asymptotically small values. This is especially true for the eigenfunctions that converge much more slowly than the eigenvalues. It should be noted that this ill-conditioning is an unavoidable feature of the numerical solution, when finite-difference schemes are used to compute the derivatives inside the inner layer. We have solved this latter problem by developing a multi-precision algorithm that relies on the Advanpix Multi-precision Computing Toolbox for MATLAB ${ }^{20}$ for arbitrary machine precision calculations and that solves the eigenvalue problem related to the finite difference scheme described above with a generalized Schur decomposition ${ }^{21,69}$ (or QZ decomposition).

In all the results that follow, the dependence of the scalings on both $\delta$ and stability parameter $\Delta^{\prime}$ introduced in Ref. 13 has been explicitly verified by direct numerical calculation. To this purpose, the scaling dependence on $\delta$ has been obtained by "defining" and thus evaluating $\delta$ as the distance of the maxima of $\psi_{1}^{\prime \prime \prime}$ (i.e., of the inflection point of the perturbed $z$-current component) from $x=0$. The one on $\Delta^{\prime}$ in the constant- $\psi$ limit relies on the estimation $\Delta^{\prime}=2 c_{1} / c_{0}$, where $c_{0} \equiv \psi_{1}(0)$ and $c_{1} \equiv \max _{(x)}\left\{\psi_{1}^{\prime}\right\}$. We will show that these definitions allow the correct estimation of the scalings already known from analytical boundary layer calculations where available (the analytical motivation behind these choices will be discussed in detail in a future paper). Among the reconnection regimes here considered, only in the viscous-resistive regime, the scalings of $\delta$ were already available from theoretical estimations ${ }^{22}$ and had been numerically confirmed. ${ }^{2}$ In the small and large wavelength limits of the warm-resistive case, they had instead been analytically obtained in Ref. 23 with heuristic arguments applied to boundary layer results in the warm-collisionless regime.

\section{A. Equilibrium choice and $\Delta^{\prime}$}

In this article, we study the (in)stability of planar current sheets $J_{z}(x, y)$ of extension $L$ along $y$ and thickness $a$ along the shear coordinate $x$, which have a single resonant point, say at $x=0$. This leads to

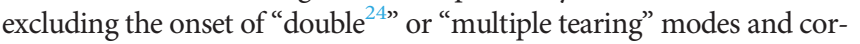
responds to a magnetic shear field that has a single zero with respect to the $x$ variable.

Although different magnetic equilibrium profiles can be considered, which are compatible with this choice, we focus here on "Grasso's equilibrium," first proposed in Ref. 25,

$$
\psi_{0}=\frac{a B_{c s}}{2 \cosh ^{2}(x / a)} .
$$

The above expression is in dimensional units. It becomes $\psi_{0}=B_{c s} /\left(2 \cosh ^{2}(x)\right)$ when normalized as in Sec. II.

We recall that in a slab geometry, the analytical form of the equilibrium profile modifies the value at fixed $k$ of the $\Delta^{\prime}$ instability parameter since the definition of the latter,

$$
\Delta^{\prime} \equiv \lim _{\varepsilon \rightarrow 0} \frac{\psi_{\text {out }}^{\prime}(\varepsilon)-\psi_{\text {out }}^{\prime}(-\varepsilon)}{\psi_{\text {out }}(0)},
$$

depends on the solution $\psi_{\text {out }}$ of the linear problem in the "outer" boundary layer domain. This corresponds to the $\mathbf{J} \times \mathbf{B}=0$ equilibrium force condition in the momentum equation (1) once linearized,

$$
\left[\psi_{\text {out }}, \psi_{0}^{\prime \prime}\right]+\left[\psi_{0}, \nabla^{2} \psi_{\text {out }}\right]=0,
$$

which is equivalent to

$$
\psi_{\text {out }}^{\prime \prime}=\left(k^{2}+\frac{\psi_{0}^{\prime \prime \prime}}{\psi_{0}^{\prime}}\right) \psi_{\text {out }} .
$$

This leads to an analytical dependence $\Delta^{\prime}=\Delta^{\prime}\left(k a ; \psi_{0}\right)$, which is here taken to be a monotone decreasing function of $k a$ (in dimensional units). Since for each equilibrium choice the interval of values of $k$ allowed for a continuous spectrum of unstable tearing modes is fixed by the condition $\Delta^{\prime}(k a)>0$, the interval of unstable wave-vectors is the interval $\left(0, k_{m g}\right)$, where $k=0$ and $k=k_{m g}$ are marginally stable modes for which $\Delta^{\prime}=0$. In the presence of a continuum spectrum of wave-vectors, the large wavelength limit $k \rightarrow 0$ and the small wavelength limit $k \rightarrow k_{m g}$ of the dispersion relation correspond to the large- $\Delta^{\prime}$ or internal-kink regime ${ }^{14,15}$ and to the small- $\Delta^{\prime}$ or constant- $\psi$ regime $^{13}$ (cf. also Fig. 1), respectively. 


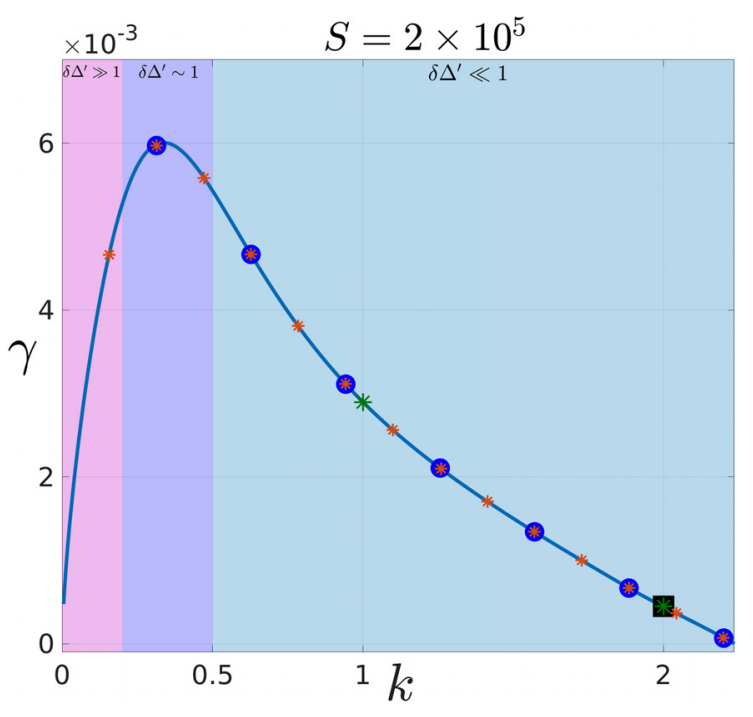

FIG. 1. The continuous line shows the dependence of $\gamma$ on $k a$ in the continuous interval $\left[0, k_{m g} a\right]$, for tearing modes due to resistivity $\left(S^{-1}=5 \times 10^{-6}\right)$. Dots, stars, and squares correspond instead to the discrete modes that can be destabilized when $L / a=\pi$ (black square), $2 \pi$ (green star), 20 (blue dots), and 40 (red stars), respectively, The largest oscillation number corresponds to $\left.\mid k_{m g} L /(2 \pi)\right\rceil$, where " $\mid .$.$\rceil " means the "integer part of." Here, k_{m g} a=\sqrt{5}$ is fixed by the equilibrium profile (6), whereas, for comparison, $k_{m g} a=1$ in the case of Harris pinch equilibrium.

In this regard, equilibrium (6), which has $k_{m g} a=\sqrt{5}$, was introduced because at fixed $k$, it provides comparably higher values of $\Delta^{\prime}$ than, for example, the $\psi_{0}=\cos (x / a)$ profile in an interval with $L_{x}=2 \pi$. In this sense, it is particularly useful for the numerical modeling of large- $\Delta^{\prime}$ modes, both for the linear (i.e., scaling analysis) and nonlinear dynamics. It must be noted that this equilibrium is periodic in $x$ only in the formal limit $L_{x} / a \rightarrow \infty$. Therefore, the periodic conditions in $x$, required by the present version of the code (they can be in principle relaxed in the numerical scheme), are granted by taking a sufficiently large interval $L_{x}$. For example, for a choice $L_{x}=4 \pi a$, which is typical for numerical simulations of tearing modes in numerical boxes with aspect ratios of order of unity, $\psi_{0}( \pm 2 \pi) \simeq 7 \times 10^{-6}$. The numerical error introduced in this way is shown to be negligible by the results presented later. We note, however, that a slightly modified version of equilibrium (6) has been also considered in Ref. 26, where constant values have been added to the first terms of the Fourier expansion in $x$ of $\psi_{0}$, in order to ameliorate the convergence of its numerical Fourier series in boxes with finite values of $L_{x} / a$.

\section{GENERAL FEATURES OF THE LINEAR CURRENT SHEET STABILITY}

The linear study of reconnecting instabilities presents a difficult analytical challenge when more than one non-ideal parameter is included in the generalized Ohm's law, even in the slab geometry approximation of the RMHD model that we consider here. The main reason is due to the fact that the boundary layer analysis, first used to address this problem in the resistive regime in the seminal paper by Furth, Killeen, and Rosenbluth ${ }^{13}$ (FKR, hereafter), often requires a multiple boundary layer approach when more microscopic scales are introduced, with each layer being related to a different non-ideal parameter. Indeed, it is not trivial to identify the characteristic scale lengths delimiting the size of each boundary layer since they require close inspection of the differential equations involved and since their scaling with the non-ideal parameters depend on a priori orderings that need to be verified once the solution has been obtained. The need to perform a multiple boundary layer calculation has been shown by Pegoraro and Schep in the so-called semi-collisional regime. ${ }^{18}$ In that article, the linear problem for tearing modes in the presence of both resistivity and electron (and ion) temperature effects has been solved analytically in Fourier space. This multiple boundary layer technique has, then, been adopted in other regimes: in the semi-collisional internal-kink instability, ${ }^{17}$ in viscous-resistive reconnection, ${ }^{22}$ where both finite resistivity and ion viscosity influence the growth rate, and in collisionless regimes, ${ }^{27}$ where reconnection is ruled by electron inertia and Finite-Larmor-Radius effects.

These analytical estimates have provided for the years to follow the basis of several studies in which the scaling of the reconnection rate plays a fundamental role also for the interpretation of nonlinear simulation results. Relatively few works have undertaken the systematic numerical study of the problem and the verification of the few available theoretical estimates, especially for tearing on single resonant surfaces.

At the same time, it is worth recalling that it is sometimes possible to obtain the estimates of the growth rate and of the layer width by combining the boundary layer approach with some ansatz about the ordering of terms and scales in the linear equations, which are essentially based on dimensional estimates and whose consistency must be verified a posteriori. This is what we hereafter refer to as the "heuristic approach." Below, we are going to provide an example in the purely resistive regime (and in the viscous-resistive regime, in Sec. V).

Aiming at reviewing some qualitative, general features of the current sheet stability, let us now proceed with some dimensional estimates.

Consider first a current sheet of intensity $J_{0}$ in an MHD hydrogen-type plasma with (ion) density $n_{0}$ and mass $m$. The "natural timescale" that we can build with these parameters, irrespectively of the width of the sheet, is

$$
\tau_{0} \sim\left(\frac{m n_{0}}{4 \pi}\right)^{1 / 2}\left(\frac{c}{J_{0}}\right) .
$$

It must be noted that only the current density amplitude, $J_{0}$, enters in this definition. In the geometry we have chosen, it can be specialized further so to make explicit its dependence on a characteristic length and on a magnetic field intensity by using $J_{0} \sim B_{c s} / a$; in this case, $\tau_{0}$ becomes the transition time of a shear Alfvén wave across the distance $a$. Notice, however that, unless additional hypothesis is made, in general, $B_{c s} \neq B_{0}$ since the validity of the reduced MHD equations only requires that the relevant time-scales are longer than the inverse total cyclotron frequency.

For illustrative purposes, let us now proceed by considering for simplicity the case of the purely resistive tearing. By restoring the dimensions in Eqs. (3) and (4), one has

$$
\begin{gathered}
\gamma \psi_{1}=i k \psi_{0}^{\prime} \hat{\varphi}_{1}+\eta_{m}\left(\psi_{1}^{\prime \prime}-k^{2} \psi_{1}\right), \\
\gamma 4 \pi n_{0} m\left(\hat{\varphi}_{1}^{\prime \prime}-k^{2} \hat{\varphi}_{1}\right)=i k \psi_{0}^{\prime}\left(\psi_{1}^{\prime \prime}-k^{2} \psi_{1}\right)-i k \psi_{0}^{\prime \prime \prime} \psi_{1},
\end{gathered}
$$


where $\hat{\varphi} \equiv c \phi / B_{\text {tot }}^{0}$. Here, $\phi$ is the electrostatic potential and $B_{\text {tot }}^{0}$ the modulus of the total equilibrium magnetic field, which, in the strong guide field limit having $B_{z}^{0} \gg B_{c s}$, is $B_{t o t}^{0} \simeq B_{z}^{0}=B_{0}$. Note that, although dimensional, these equations have no explicit dependence on the reference magnetic field $B_{0}$, which has been incorporated in the quantity $\hat{\varphi}$, the stream function of the fluid.

When we consider the solution of Eqs. (11) and (12) at a distance from the neutral line of the order of the reconnecting layer width $\delta$, we can neglect $k^{2}$ with respect to the second order derivative in $x$ of the perturbed quantities. We can also approximate $\left.\left.\psi_{0}^{\prime}\right|_{x \simeq \delta} \sim \delta \psi_{0}^{\prime \prime}\right|_{x \simeq_{\delta}}$ $\simeq 4 \pi J_{0} \delta / c$ and neglect the $\psi_{0}^{\prime \prime \prime}$ term. By ordering $\psi_{1}^{\prime \prime} / \psi_{1} \sim 1 / \delta^{2}$, we, then, obtain the two time scales

$$
\gamma \sim \frac{k \delta}{\tau_{0}} \text { and } \quad \gamma \sim \frac{\eta_{m}}{\delta^{2}}
$$

The former is essentially the shear-Alfvén wave dispersion relation at the scale $x \sim \delta$, it is related to the natural timescale $\tau_{0}$, and it does not depend explicitly on the current sheet width $a$ nor on the characteristic system length $L$.

The second timescale is the resistive diffusion time along $x$ over the scale $\delta$.

The above estimate is valid in the large $\Delta^{\prime}$ limit, that is, as long as $\Delta^{\prime} \delta \gg 1$.

In the small- $\Delta^{\prime}$ limit, one must assume $\psi_{1}^{\prime} / \psi_{1} \sim \Delta^{\prime}$ and $\psi_{1}^{\prime \prime} / \psi_{1} \sim \Delta^{\prime} / \delta$. One, then, has the two conditions

$$
\gamma \sim \frac{k \delta\left(\Delta^{\prime} \delta\right)^{1 / 2}}{\tau_{0}} \text { and } \quad \gamma \sim \frac{\eta_{m} \Delta^{\prime}}{\delta} .
$$

We have, thus, heuristically obtained the following scalings in the large- (label $L D$ ) and small- $\Delta^{\prime}$ (label $S D$ ) limits, respectively:

$$
\begin{gathered}
\gamma_{L D} \sim \frac{k \delta_{L D}}{\tau_{0}}, \quad \delta_{L D} \sim\left(\tau_{0} k^{-1} \eta_{m}\right)^{1 / 3}, \\
\gamma_{S D} \sim \frac{k \delta_{S D}\left(\Delta^{\prime} \delta_{S D}\right)^{1 / 2}}{\tau_{0}}, \quad \delta_{S D} \sim\left(\tau_{0} k^{-1} \eta_{m}\right)^{2 / 5} \Delta^{\prime 1 / 5} .
\end{gathered}
$$

Note that by treating the wavenumber as a parameter, there is no explicit dependence on the sheet length $L$. Moreover, the dependence on the scale $a$ occurs only for the small- $\Delta^{\prime}$ solution via the instability parameter $\Delta^{\prime}(k a)$, which fixes the range of values of $k a \in\left(0, k_{m g} a\right)$ allowed for the unstable modes.

Scalings [(15) and (16)], then, define the two ranges of the spectrum of unstable modes, which meet at a wavenumber such that $\delta_{L D} \sim \delta_{S D} \sim \Delta^{\prime-1}$. This situation is illustrated in Fig. 1, where the dependence of $\gamma$ is represented with respect to both a continuum spectrum and a discrete spectrum in $k$; the latter is given for different values of $L / a$. One observes that the wavenumber at which the two ranges meet corresponds to the most unstable mode if $L / a$ is sufficiently large.

The scaling of this fastest growing mode depends on the magnetic equilibrium profile, and its calculation generally requires a numerical approach even if estimates based on the algebraic scaling of $\Delta^{\prime}(k a)$ for small values of $k a$ can be given from the small- and large$\Delta^{\prime}$ limits of the dispersion relation. ${ }^{29,30}$

The scaling of the fastest growing mode was already addressed in a series of papers by Cross and Van Hoven, ${ }^{31-34}$ which were mainly focused on applications to astrophysical plasmas. In these papers, the typical aspect ratio of the current sheet is taken to be very large, so the approximation $L / a \rightarrow \infty$ can be adopted. A paradigmatic example, already considered by Dungey in his first formalization of the notion of reconnection, ${ }^{35,36}$ is provided by the terrestrial magnetotail. Its instability to reconnecting modes in a continuous spectrum of wavelengths has been addressed in a kinetic framework in Ref. 37 and then by considering resistive slab tearing modes in Refs. 32 and 38. Other astrophysical applications of tearing theory to large aspect ratio current sheets have been, then, considered for coronal mass ejections (see Ref. 39-42), whose occurrence close to neutral magnetic points noted by Giovanelli ${ }^{43}$ had indeed motivated Hoyle ${ }^{44}$ and Dungey ${ }^{35,36}$ to explore and formalize the mechanism that would have been later dubbed as magnetic reconnection. ${ }^{45}$ In particular, as noted by Velli and Hood, ${ }^{41}$ an aspect ratio of the order of $L / a \sim 20$ is typically sufficient to allow the instability of modes with a wave-vector close enough to that of the fastest growing mode as computed by considering a continuous spectrum (cf. Fig. 1).

In the numerical results that follow, however, the fastest mode has been identified by selecting, for each fixed couple of non-ideal parameters at play, the wave number that displayed the maximum growth rate after a scan over about 40 modes in the $(0, \sqrt{5})$ interval of the (normalized) $k$ variable.

Although only the small- $\Delta^{\prime}$ and fastest growing mode wavelength limits are physically relevant to the stability of a planar current sheet (cf. discussion in Sec. IX A), it must be noted that the slab tearing analysis in the $k \rightarrow 0$ limit is formally equivalent to that of the internal kink mode in large aspect ratio tokamaks. ${ }^{14,15}$ Because of this, in the following, we will always note in each reconnection regime the scalings in the large- $\Delta^{\prime}$ limit too.

\section{VISCOUS-RESISTIVE REGIME $\left(R^{-1}, S^{-1}\right)$}

We here identify as "viscous-resistive" the regime in which we can set $d_{e}=\rho_{s}=0$ in Eqs. (1) and (2). The eigen-matrix coefficients in Eq. (5) take, then, the form

$$
\begin{aligned}
& \mathscr{A}=\frac{\partial^{2}}{\partial x^{2}}-k^{2}, \quad \mathscr{D}=1, \quad \mathscr{L}_{1}=S^{-1} \mathscr{A}, \quad \mathscr{L}_{2}=-k \psi_{0}^{\prime}, \\
& \mathscr{L}_{3}=k \psi_{0}^{\prime} \mathscr{A}-i k \psi_{0}^{\prime \prime \prime}, \\
& \mathscr{L}_{4}=i R^{-1} \mathscr{E}, \text { and } \mathscr{E}=\frac{\partial^{4}}{\partial x^{4}}-2 k^{2} \frac{\partial^{2}}{\partial x^{2}}+k^{4} .
\end{aligned}
$$

Although linear tearing modes in this regime have been already extensively studied in the literature (see Refs. 1, 2, 22, 46, and 47), they are considered here for two reasons. The first one is that previous studies in this regime chose different equilibria from (6). The second one is that comparison with previous results that do not depend on the choice of the equilibrium profile (notably the results in the constant- $\psi$ or small- $\Delta^{\prime}$ limit) provides a benchmark test for the newly developed eigensolver.

We have recovered the well-known scaling laws both in the purely resistive $e^{13,14,32}$ regime,

$$
\begin{aligned}
\gamma_{L D} \sim k^{2 / 3} S^{-1 / 3}, & \delta_{L D} \sim k^{-1 / 3} S^{-1 / 3}, \\
\gamma_{S D} \sim k^{2 / 5} \Delta^{/ 4 / 5} S^{-3 / 5}, & \delta_{S D} \sim k^{-2 / 5} \Delta^{1 / 5} S^{-2 / 5},
\end{aligned}
$$

and in the viscous-resistive regime, ${ }^{22}$ 


$$
\begin{array}{cc}
\gamma_{L D} \sim k^{2 / 3} R^{1 / 3} S^{-2 / 3}, & \delta_{L D} \sim k^{-1 / 3} R^{-1 / 6} S^{-1 / 6}, \\
\gamma_{S D} \sim k^{1 / 3} \Delta^{\prime} R^{1 / 6} S^{-5 / 6}, & \delta_{S D} \sim k^{-1 / 3} R^{-1 / 6} S^{-1 / 6},
\end{array}
$$

where the subscripts "LD" and "SD" label the large- $\Delta^{\prime}$ or large wavelength limit $k a \rightarrow 0^{+}$and the small- $\Delta^{\prime}$ or small wavelength $k a \rightarrow$ $\left(k_{m g} a\right)^{-}$limit of the dispersion relation, respectively. Note that the reconnecting layer width in the viscous-resistive regime has the same scaling in both the large- and small- $\Delta^{\prime}$ limits [Eqs. (19) and (20)]. Scalings [(17) and (18)] correspond to (15) and (16), respectively, once the latter are normalized as specified in Sec. II.

These benchmark results are shown in the right frames of Fig. 2. For the growth rates, these scalings had been already numerically verified in the past, for example, in Refs. 1, 46, and 47, although with a different magnetic equilibrium (which does not affect these results). It is, however, in the more recent work by Tenerani et al. ${ }^{2}$ that a more extensive numerical study has been performed also for the scaling of the reconnecting layer width and in all wavelength limits of the slab dispersion relation, with a different eigensolver than the one used here.

The scalings [(17)-(20)] can be estimated by means of the heuristic-type argument outlined in Sec. IV. A relation is obtained by balancing the dominant terms of Eq. (4), which, by assuming $\varphi_{1}^{(N)}$ $\sim \varphi_{1} / \delta^{N}$ (with ${ }^{(N)}$ expressing the $N$ th-order derivative with respect to $x$ ), we write as $\gamma \varphi_{1} / \delta^{2} \sim i k \delta \psi_{1}^{\prime \prime}+R^{-1} \varphi_{1} / \delta^{4}$. Using the latter and the estimates obtained from (3) so to eliminate $\varphi_{1}$ and $\psi_{1}^{\prime \prime}$ in terms of $\psi_{1}$ (that is, $\varphi_{1} \sim-i \gamma \psi_{1} /(k \delta)$ and $\psi_{1}^{\prime \prime} \sim \gamma S \psi_{1}$ ), we get the qualitative expression as follows:

$$
\gamma \sim R^{-1} \delta^{-2}-k^{2} \delta^{4} S
$$

Combination of (21) with the relations obtained from Ohm's law (second relation of (13) and (14)) yields two polynomial relations for $\gamma$ (or $\delta$ ) whose solution gives the sought scalings. Equations (19) and (20) are obtained by assuming the $R^{-1}$-related terms to be dominant, which, by developing the algebra, is seen to correspond to $R^{-1} \gg S^{-1}$ for $\Delta^{\prime} \delta_{L D} \gg 1$ and to $\gamma \sim\left(S^{-1} \Delta^{\prime} / \delta_{S D}\right) \ll R^{-1} \delta_{S D}^{-2}$ for $\Delta^{\prime} \delta_{S D} \ll 1$. Using (20), the latter condition a posteriori means $R^{-1} \gg S^{-7 / 5}\left(k^{-1 / 3} \Delta^{\prime}\right)^{6 / 5}$. Leaving $k$ unordered with respect to $R$ and $S$, one so recovers the condition $R^{-1} \gg S^{-7 / 5}$, already evidenced by Bian and Vekstein ${ }^{48}$ from boundary layer calculations in a problem of forced reconnection. Using instead the upper limit of the $\Delta^{\prime} \delta_{S D} \lesssim 1$ condition, that is, of $k^{-1 / 3} \Delta^{\prime} \leq R^{1 / 6} S^{1 / 6}$, the $R^{-1} \gg S^{-1}$ ordering is re-obtained. Equations (17) and (18) are obtained in the opposite limits when $S^{-1}$-related terms are dominant. The condition $R^{-1} \gg S^{-1}$ in the large- $\Delta^{\prime}$ limit is, therefore, more restrictive and can be generally assumed as required for the occurrence of the viscous-resistive regime, when a continuous variation of $k$ is assumed in a slab geometry.

A further scaling, which is of physical interest in a large aspect ratio current sheet, is that of the mode with the highest growth rate. ${ }^{13}$ The scalings of this mode occurring at $\Delta^{\prime} \delta \sim 1$ do depend on the choice of the equilibrium profile, ${ }^{29}$ as it had been already numerically found by Cross and Van Hoven. ${ }^{31}$ Previous studies of this mode in the viscous-resistive regime have focussed ${ }^{2}$ on a Harris-pinch equilibrium. ${ }^{28}$ In Ref. 29, however, general formulas for the maximum growth rate for a general equilibrium profile have been proposed for the collisionless, purely resistive and viscous-resistive regimes [see Eqs. (23)-(25) therein]: these scalings can be estimated by equating $\gamma_{S D}\left(k_{M}\right)=\gamma_{L D}\left(k_{M}\right)=\gamma_{M}$, while assuming $\Delta^{\prime}(k) \sim k^{-p}$ for $k \ll 1$. These formulas agree with the results obtained for a Harris-pinch profile $^{2,13,49}$ (case $p=1$ ), and they have been verified also for equilibrium (6), indirectly in the warm collisionless case in Ref. 29 (by comparison with previous results by Comisso et al..$^{50}$ ) and directly with another numerical solver in the purely resistive, inviscid regime in Ref. 30.
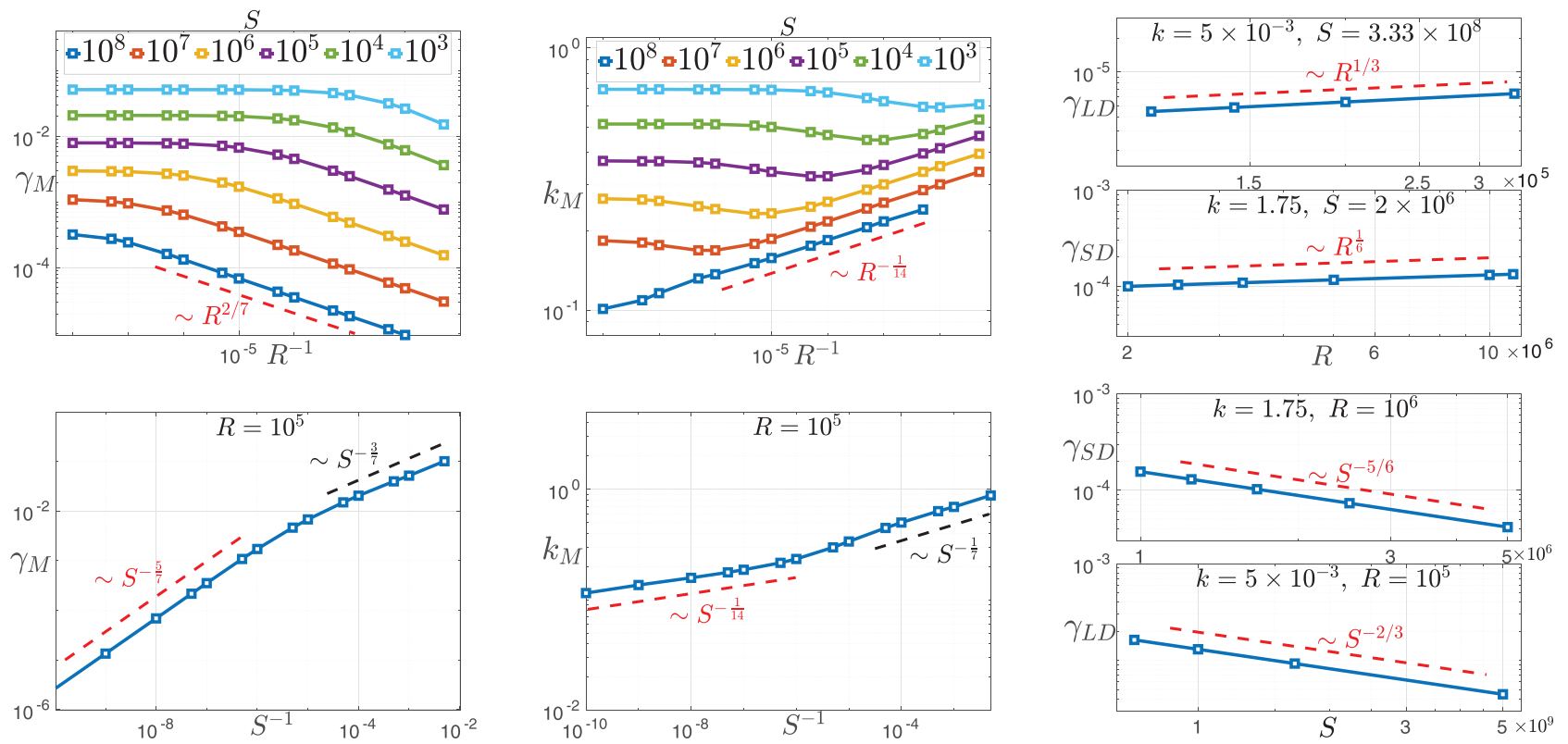

FIG. 2. Scaling laws in the visco-resistive regime of Sec. $V$ (dots are numerically obtained values). Left frames: growth rate of the fastest growing mode $\gamma_{M}$ as a function of $R$ (top) and of $S$ (bottom). Center frames: wavenumber of the fastest growing mode $k_{M}$ as a function of $R$ (top) and $S$ (bottom). Right frames: growth rates in the large- $\Delta^{\prime}$ and small- $\Delta^{\prime}$ limits (labels $\gamma_{L D}$ and $\gamma_{S D}$, respectively) as a function of $R$ and $S$. 
For convenience, we rewrite here these general scalings for the fastest growing mode $\Delta^{\prime} \delta \sim 1$ by including also the corresponding estimates for $k_{M}$ and $\delta_{M}$ (they can be, then, easily rewritten in terms of Prandtl number $P \equiv \nu / \eta=S / R$ ),

$$
\begin{gathered}
\begin{array}{c}
\text { Viscous }- \text { Resistive } \\
\text { regime }(P \gg 1) \\
\text { for } \lim _{k \rightarrow 0} \Delta^{\prime} \sim k^{-p}
\end{array} \quad\left\{\begin{array}{l}
\gamma_{M} \sim R^{\frac{p}{1+3 p}} S^{-\frac{1+2 p}{1+3 p}}, \\
k_{M} \sim\left(R^{-1 / 2} S^{-1 / 2}\right)^{\frac{1}{1+3 p}} \\
\delta_{M} \sim\left(R^{-1 / 2} S^{-1 / 2}\right)^{\frac{p}{1+3 p}},
\end{array}\right. \\
\quad \begin{array}{l}
\gamma_{M} \sim S^{-\frac{1+p}{1+3 p}}, \\
k_{M} \sim S^{-\frac{1}{1+3 p}}, \\
\delta_{M} \sim S^{-\frac{p}{1+3 p}} .
\end{array}
\end{gathered}
$$

In the left and center frames of Fig. 2, we have plotted the scalings of $\gamma_{M}$ and $k_{M}$, with respect to the Reynolds and Lundquist numbers, which we have numerically obtained by solving the eigenvalue problem for equilibrium (6). The complete set of numerical results is summarized by

$$
\begin{array}{cl}
\begin{array}{c}
\text { Viscous - Resistive } \\
\text { regime }(P \gg 1)
\end{array} & \left\{\begin{array}{l}
\gamma_{M} \sim R^{2 / 7} S^{-5 / 7}, \\
k_{M} \sim R^{-1 / 14} S^{-1 / 14}, \\
\delta_{M} \sim R^{-1 / 7} S^{-1 / 7},
\end{array}\right. \\
\begin{array}{c}
\text { Resistive } \\
\text { regime }(P \ll 1)
\end{array} & \left\{\begin{array}{l}
\gamma_{M} \sim S^{-3 / 7}, \\
k_{M} \sim S^{-1 / 7}, \\
\delta_{M} \sim S^{-2 / 7} .
\end{array}\right.
\end{array}
$$

Equations (24) and (25) correspond to the analytical estimations [(22) and (23)] after the substitution $p=2$, valid for equilibrium (6). The transition to a purely resistive regime in which the effects of viscosity become negligible is qualitatively in agreement with that obtained in Ref. 2 for a Harris equilibrium, and the scalings for $\gamma_{M}$ and $k_{M}$ of (25) coincide with those already verified in Ref. 30.

Two distinct regions are visible in Fig. 2. The first one is for $R \leq S$ (see, e.g., the yellow line in the top frames of Fig. 2 for $R \leq S=10^{6}$ ). This region represents the visco-resistive case, and its scalings confirm those of Eq. (24). The second region is for $R \geq S$ (see, e.g., the yellow line of Fig. 2 for $R>S=10^{6}$ ) and corresponds to a purely resistive limit in which viscosity negligibly contributes. Its scalings are given by Eq. (25). A transition region between these two limit regimes is also visible. This region is characterized by a reversal of the slope in the scaling of $k_{M}$ with $R^{-1}$-see the top-center frame of Fig. 2, where a relative minimum of $k_{M}\left(R^{-1}\right)$ is clearly visible. No power law scaling has been measured here.

The growth rates of the fastest mode in both viscous-resistive and purely resistive regimes for equilibrium (6) can be compared by rewriting their ratio in terms of the Prandtl number: $\gamma_{M_{S}} \sim P^{2 / 7} \gamma_{M_{S, R}}$, where $\gamma_{M_{S}}$ stands for the scaling of Eq. (24) and $\gamma_{M_{S, R}}$ for that of $(25)$. This relationship indicates the intuitive role of the viscosity in slowing down the tearing growth rate as $P$ overtakes unity (cf. the values of $\gamma_{M}$ plotted in the right frames of Fig. 2). In the parameter range we have numerically explored, the limits of applicability of Eqs. (24) and (25) in terms of the value of $P$ can be quantitatively expressed as given by $P \leq 10^{-1}$ and $P \geq 10$, respectively.

\section{WARM-RESISTIVE REGIME $\left(\rho_{\boldsymbol{s}}, \mathbf{S}^{-1}\right)$}

We consider the regime in which only temperature effects and a finite collision rate dominate. That is, we can assume $R^{-1}=d_{e}=0$ in Eqs. (3) and (4) so that they become

$$
\begin{gathered}
\gamma \psi=k \rho_{s}^{2} \psi_{0}^{\prime}\left(\frac{\partial^{2}}{\partial x^{2}}-k^{2}\right) \varphi-k \psi_{0}^{\prime} \varphi+S^{-1}\left(\frac{\partial^{2}}{\partial x^{2}}-k^{2}\right) \psi, \\
\gamma\left(\frac{\partial^{2}}{\partial x^{2}}-k^{2}\right) \varphi=\left[k \psi_{0}^{\prime}\left(\frac{\partial^{2}}{\partial x^{2}}-k^{2}\right)-k \psi_{0}^{\prime \prime \prime}\right] \psi .
\end{gathered}
$$

The coefficients of the eigen-matrices of Eq. (5) are $\mathscr{A}=\frac{\partial^{2}}{\partial x^{2}}$ $-k^{2}, \mathscr{D}=1, \mathscr{L}_{1}=S^{-1} \mathscr{A}, \mathscr{L}_{2}=k \rho_{s}^{2} \psi_{0}^{\prime} \mathscr{A}-k \psi_{0}^{\prime}, \quad \mathscr{L}_{3}=k \psi_{0}^{\prime} \mathscr{A}$ $-k \psi_{0}^{\prime \prime \prime}$, and $\mathscr{L}_{4}=0$.

The linear problem in this warm-resistive regime has been first studied analytically by means of a boundary layer theory in Ref. 18 . Although in that treatment diamagnetic drift effects were also included, here we neglect them, as equilibrium density fluctuations are not retained in the model of Eqs. (3) and (4). In the same paper, the effect of finite ion Larmor radius corrections was also retained by means of a (gyro)-kinetic modeling of the ion response. ${ }^{51}$ Although ion FLR effects can be included in the model by means of Padé-type approximants $^{6,52}$ or by means of more refined approximations, ${ }^{53,54}$ which, in the collisionless limit, preserve the Hamiltonian character of the system for the arbitrary values of $\rho_{i}$, we have chosen not to include them in the present analysis. In this sense, we depart from the "semicollisional" limit, identified in the kinetic study by Drake and Lee ${ }^{19}$ through the condition $\gamma d_{e}^{2} \ll S^{-1} \ll\left(k d_{e}\right) \Delta^{\prime-1 / 2} \rho_{i}^{3 / 2}$.

The scalings given in Refs. 17 and 18 for the warm-resistive case and in Ref. 27 for the warm-collisionless case can be recovered from those discussed in this work with the substitution $\rho_{s}^{2} \rightarrow \rho_{i}^{2}+\rho_{s}^{2}$. However, the loss of symmetry between $\rho_{s}^{2}$ and $\rho_{i}^{2}$ in the warmcollisionless growth rate, which was noted in Ref. 55, requires further investigation. The warm-collisionless scalings $\left(d_{e} \neq 0, \rho_{s} \neq 0\right)$ were also obtained by Zocco and Scheckochihin, ${ }^{23}$ starting from a reduced semi-kinetic model somewhat different from the one considered in Refs. 18, 27, and 51 (see also Ref. 6), and by integrating the boundary layer equations in coordinate space.

The heuristic estimation of the tearing scalings in the presence of $\rho_{s}$ is much more difficult than in "cold" (i.e., $\rho_{s}=0$ ) regimes. However, as it happens for the cold collisionless limit with $\rho_{s}=0$ and $d_{e} \neq 0$, in which the collisionless scalings can be deduced from those of the purely resistive regime after substituting ${ }^{56} d_{e}^{2} \rightarrow S^{-1} / \gamma$, it is easy to verify that the scalings of the warm-resistive regime below can be equally recovered from those of the warm collisionless case first discussed in Ref. 27. This is also the criterion used in Ref. 23 to re-obtain the results of Refs. 17 and 18 and to deduce the corresponding scalings for the inner layer width from boundary analysis calculations in a semi-kinetic warm-collisionless regime.

The corresponding scaling laws, confirmed by the numerical solutions, are as follows:

$$
\begin{gathered}
\gamma_{L D} \sim k^{6 / 7} \rho_{s}^{4 / 7} S^{-1 / 7}, \delta_{L D} \sim k^{-4 / 7} \rho_{s}^{-5 / 7} S^{-4 / 7}, \\
\gamma_{S D} \sim\left(k \Delta^{\prime}\right)^{2 / 3} \rho_{s}^{2 / 3} S^{-1 / 3}, \delta_{S D} \sim k^{-2 / 3} \Delta^{\prime 1 / 3} \rho_{s}^{-2 / 3} S^{-2 / 3} .
\end{gathered}
$$

In Fig. 3, we plot the growth rates $\gamma_{S D}$ and $\gamma_{L D}$ vs different parameters $\left(k, S\right.$, and $\left.\rho_{s}\right)$ and the corresponding scaling laws of $\delta_{S D}$ and $\delta_{L D}$. The conditions of validity of these scalings are obtained from the 

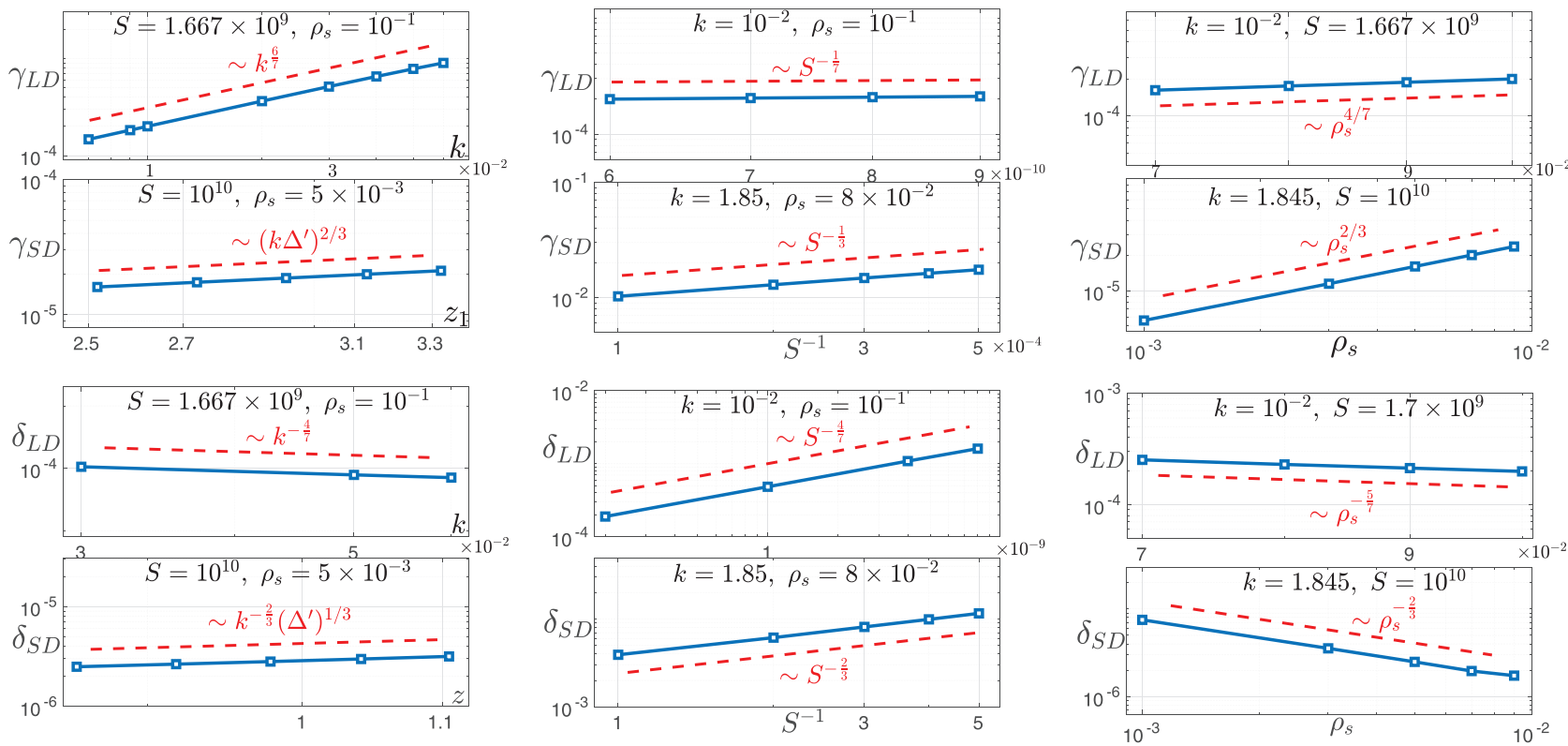

FIG. 3. Scaling laws in the warm-resistive regime of Sec. VI (blue dots are numerically obtained values): growth rates $\gamma$ are displayed in the two upper panels, and reconnecting layer widths $\delta$ are displayed in the two lower panels. Labels $L D$ and $S D$ correspond to the large- $\Delta^{\prime}$ and small- $\Delta^{\prime}$ limits, respectively. In left figures (SD case), the abscissa variables are $z=k^{-2 / 3} \Delta^{1 / 3}$ and $z_{1}=\left(k \Delta^{\prime}\right)^{2 / 3}$

normalization scales of the boundary layer analysis, which give ${ }^{18,23} \rho_{s} \gg(k S)^{1 / 3}$ in the large- $\Delta^{\prime}$ limit and $\Delta^{\prime} \ll \rho_{s}^{-1 / 7}(k S)^{2 / 7}$ in the small- $\Delta^{\prime}$ limit. Outside of this range, the effect of $\rho_{s}$ is negligible and scalings [(17) and (18)] of the purely resistive regime are recovered.

Using the same argument described in Sec. V to estimate the growth rate of the fastest growing mode, we obtain from Eqs. (28) and (29) the following scalings:

$$
\begin{gathered}
\text { Warm - Resistive } \\
\text { Regime }
\end{gathered} \quad\left\{\begin{array}{l}
\gamma_{M} \sim \rho_{s}^{5 / 8} S^{-1 / 4} \\
k_{M} \sim \rho_{s}^{1 / 16} S^{-1 / 8} \\
\delta_{M} \sim \rho_{s}^{-3 / 4} S^{-1 / 2} .
\end{array}\right.
$$

These are confirmed by numerical results. Figure 4 shows the scalings of $\gamma_{M}$ (left frame) and $k_{M}$ (right frame) vs the different parameters. Here, again, two limit regions can be recognized where scalings (25) and (30), respectively, hold. In the top-left frame of Fig. 4, we see that, when $S \geq 10^{6}$, the scaling of $\gamma_{M} \propto \rho_{s}^{5 / 8}$ agrees with Eq. (30) for all chosen values of $\rho_{s}$. For the values of $S \leqslant 10^{6}$, we recognize a threshold value for the ion sound Larmor radius $\tilde{\rho}_{s}$ above which we recover the semi-collisional regime of Eq. (30). When $\rho_{s}<\tilde{\rho}_{s}$, the scaling of $\gamma_{M}$ becomes independent of $\rho_{s}$. In the bottom left frame of Fig. 4, when $S>\tilde{S}$, we recover the scaling $\gamma_{M} \sim S^{-1 / 4}$ of Eq. (30) (see, e.g., the blue line in the bottom-left frame of Fig. 4, where we can identify $\tilde{S}=10^{7}$ for $\rho_{s}=10^{-2}$ ). The second limit regime (purely resistive) governed by Eq. (25) corresponds to $\gamma_{M} \sim S^{-3 / 7}$ and is observed when $S<\tilde{S}$ (see again, e.g., the blue line in the bottom-left frame of Fig. 4 for $\rho_{s}=10^{-2}$ ). In the right frame of Fig. 4, we recognize the same features as above. Here, a threshold value of the Lundquist number $\tilde{S}$ for each value of $\rho_{s}$ is visible. For larger values of $S$, the wavenumber of the fastest growing mode follows the scaling $k_{M} \sim S^{-1 / 8}$ of the warmresistive regime given by Eq. (30), while below this threshold value, the wavenumber of the fastest growing mode is once more governed by the scaling $k_{M} \sim S^{-1 / 7}$.

The spatial profile of the eigenmodes obtained in this regime, in which $\rho_{s}$ explicitly affects the eigenvalues, perfectly agrees with its approximated analytical form provided in Ref. 18 for $\left|x / \rho_{s}\right| \ll 1$. In dimensional units and in the notation of the present article, Eq. (74) of Ref. 18 reads

$$
J_{z}(x / a)=-\psi_{1}^{\prime \prime}(x / a) \simeq-\frac{2 c_{1}}{\pi}\left(\frac{\rho_{s}}{a}\right) \frac{\left(\gamma \tau_{A} S^{-1}\right)^{1 / 2}}{k^{2} \rho_{s}^{2}(x / a)^{2}+\gamma \tau_{A} S^{-1}},
$$

as the following correspondence between the variables of the aforementioned reference and the ones we use here in the slab-geometry limit holds: $\hat{s}=1$ (we have arbitrarily chosen the magnetic shear parameter $\hat{s}$ of the tokamak to be equal to unity in the slab geometry approximation), $\varepsilon=S^{-1}, m=k a, \mu=1$ (isothermal limit), $g_{e}=1$ (uniform equilibrium density), $\tau b=\left(k \rho_{s}\right)^{2}, z_{\rho}^{2}=1 /\left(k \rho_{s}\right)^{2}$, $z_{R}^{2}=-\gamma \tau_{A} S /(k a)^{2}, z_{A}^{2}=-\left(\gamma \tau_{A}\right)^{-2}, \quad$ and $\quad z_{j}^{2}=\left(g_{e} z_{R}^{2} z_{A}^{2}\right)^{1 / 2}$ $=\left(\gamma \tau_{A} k a\right)^{-1 / 2} S^{1 / 2}$. In Fig. 5, the spatial profile of (31) is compared to that numerically obtained from the eigenmode $\psi_{1}$ in the interval $-\rho_{s} \leq x \leq \rho_{s}$ for the same set of parameters of the center-bottom frame of Fig. 3; here, $\delta \simeq 3 \times 10^{-4}$.

Finally, the scalings of Eq. (30) can be generalized to an arbitrary equilibrium profile. Proceeding as before, we obtain

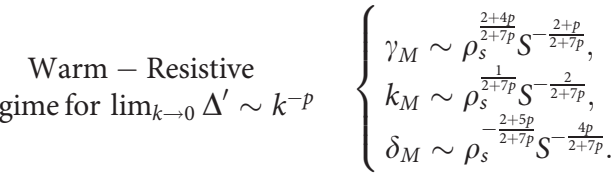



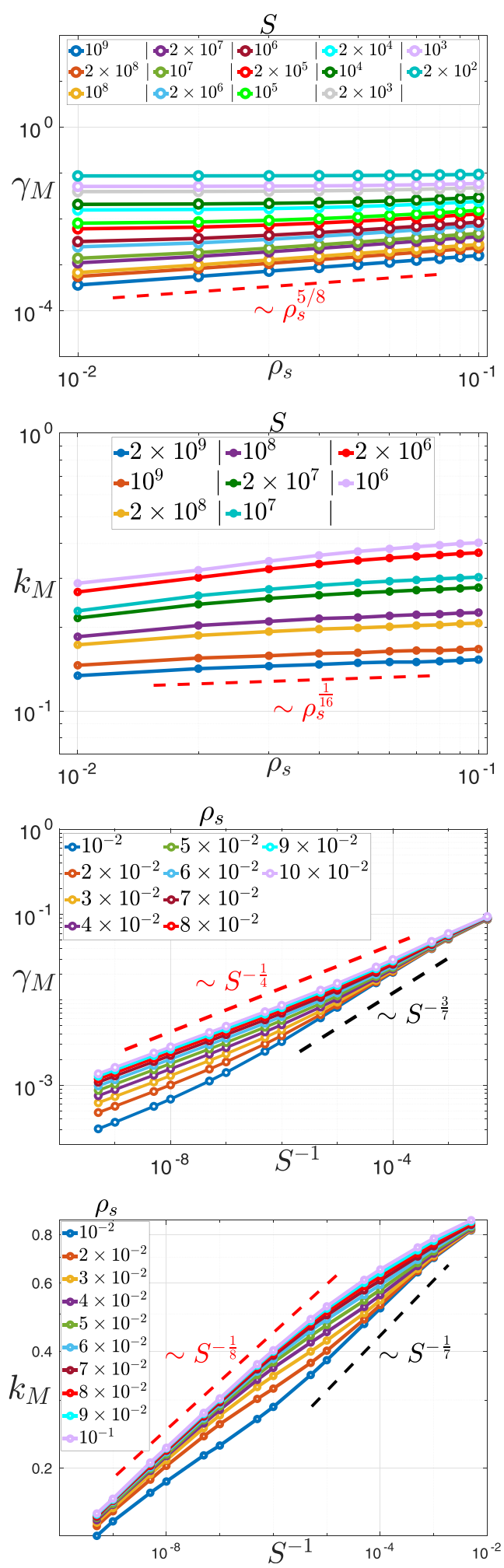

FIG. 4. Scaling laws of the fastest growing mode $\left(\Delta^{\prime} \delta \sim 1\right)$ in the warm-resistive regime (Sec. VI).

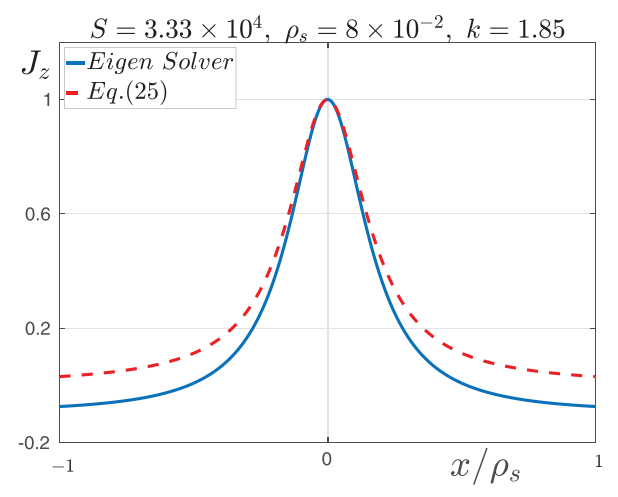

FIG. 5. Perturbed current density profiles normalized to their maximum value, compared in the $-\rho_{s} \leq x \leq \rho_{s}$ interval: the blue curve represents the current density numerically computed with the eigensolver, while the red one is obtained using Eq. (31), formally valid for $|x| \ll \rho_{s}$. Here, the inner layer width is $\delta \simeq 3 \times 10^{-4}$.

\section{WARM-INERTIAL REGIME $\left(\rho_{s}, \boldsymbol{d}_{\mathrm{e}}\right)$}

This regime differs from the previous one in that a finite electron inertia replaces a finite resistivity as the mechanism that allows reconnection by violating the ideal Lagrangian invariance of $\psi$. We, then, assume $R^{-1}=S^{-1}=0$ in Eqs. (3) and (4), and the coefficients of Eq. (5) become $\mathscr{A}=\frac{\partial^{2}}{\partial x^{2}}-k^{2}, \quad \mathscr{D}=1+k^{2} d_{e}^{2}-d_{e}^{2} \frac{\partial^{2}}{\partial x^{2}}, \quad \mathscr{L}_{1}=\mathscr{L}_{4}$ $=0, \mathscr{L}_{2}=k \rho_{s}^{2} \psi_{0}^{\prime} \mathscr{A}-k F_{0}^{\prime}, \mathscr{L}_{3}=k \psi_{0}^{\prime} \mathscr{A}-i k \psi_{0}^{\prime \prime \prime}$.

Scalings of the growth rate in this regime have been first obtained by Porcelli ${ }^{27}$ and had been provided for the layer width $\delta$ by Zocco and Schekochihin. ${ }^{23}$ For the fastest growing mode, they had been first numerically obtained by Comisso et al..$^{50}$ for the same equilibrium (6) we consider here, and they had been generalized to different equilibria in Ref. 29.

As mentioned in Sec. VI, scalings in this regime mirror those of the warm-resistive case previous the substitution $S^{-1} \rightarrow \gamma d_{e}^{2}$.

In the regime where both $d_{e}$ and $\rho_{s}$ contribute, which is delimited by the condition $\rho_{s} \geq d_{e}$, we rewrite below all the relevant scalings, whereas we present in Fig. 6 the numerical results for the small- and large- $\Delta^{\prime}$ regimes, only, since the fastest growing mode with $p=2$ has been already studied,

$$
\begin{gathered}
\gamma_{L D} \sim k \rho_{s}^{2 / 3} d_{e}^{1 / 3}, \quad \delta_{L D} \sim \rho_{s}^{-1 / 3} d_{e}^{4 / 3}, \\
\gamma_{S D} \sim k \Delta^{\prime} \rho_{s} d_{e}, \quad \delta_{S D} \sim \Delta^{\prime} d_{e}^{2}, \\
\text { Warm - Inertial } \\
\text { Regime for } \lim _{k \rightarrow 0} \Delta^{\prime} \sim k^{-p}\left\{\begin{array}{l}
\gamma_{M} \sim \rho_{s}^{\frac{1+2 p}{3 p}} d_{e}^{\frac{2+p}{3 p}}, \\
k_{M} \sim \rho_{s}^{\frac{1}{3 p}} d_{e}^{\frac{2}{3 p}} \\
\delta_{M} \sim \rho_{s}^{-1 / 3} d_{e}^{4 / 3} .
\end{array}\right.
\end{gathered}
$$

Note, in the large- $\Delta^{\prime}$ limit, the localization of the reconnecting layer in a scale $\delta_{L D}$ smaller than $d_{e}$ because of $\rho_{s}>d_{e}$ and, in the small- $\Delta^{\prime}$ limit, the independence of $\delta_{S D}$ from $\rho_{s}$.

It is well known, however, that for $d_{e} \gg \rho_{s}$, the purely inertial (i.e., cold collisionless) regime is entered. Its scalings in the large- and small- $\Delta^{\prime}$ limits have been provided in Refs. 27 and 56 and in Refs. 16, 19 , and 56 , respectively,

$$
\gamma_{L D} \sim k d_{e}, \quad \delta_{L D} \sim d_{e}
$$



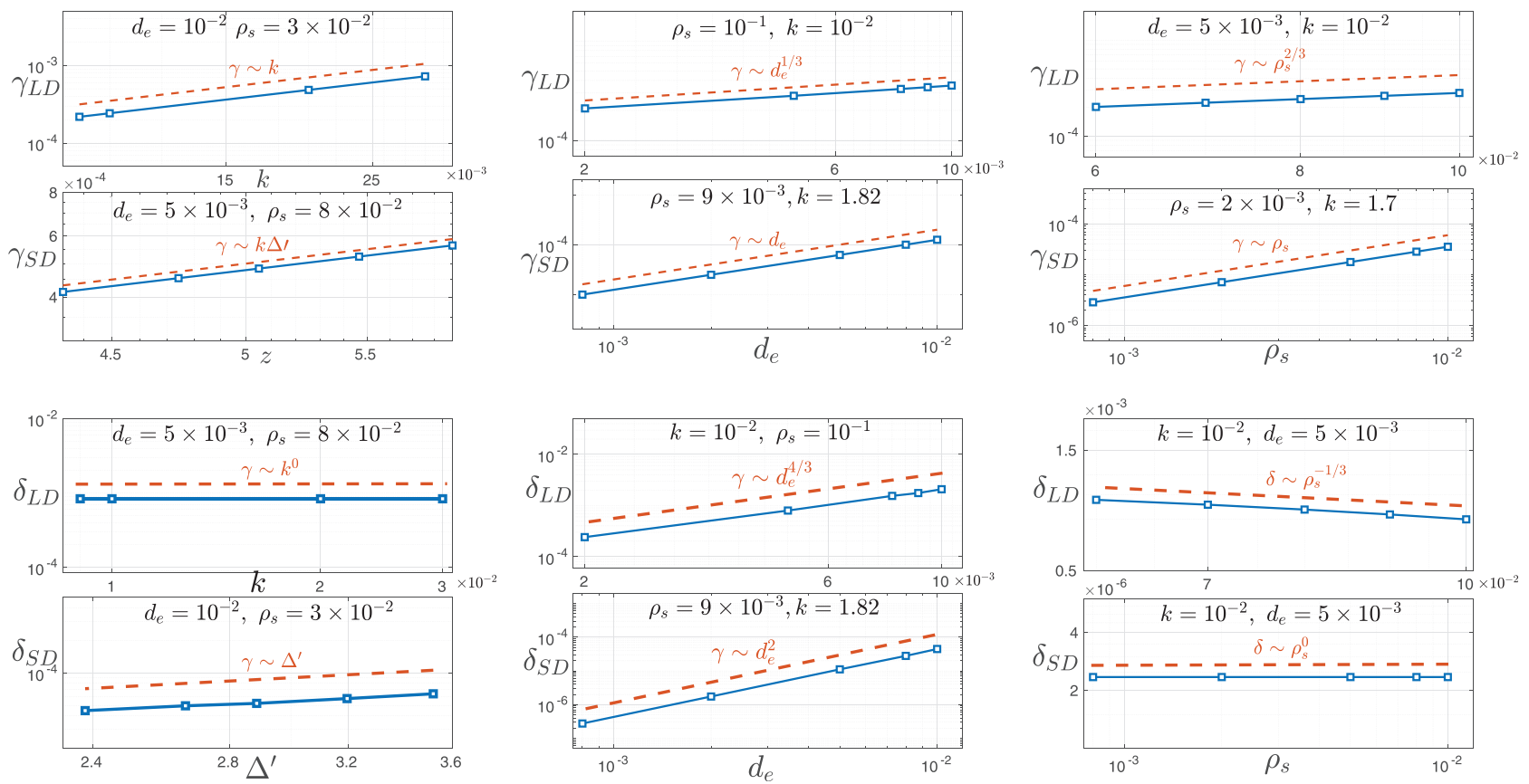

FIG. 6. Scaling laws in the warm-inertial regime of Sec. VII (blue dots are numerically obtained values): growth rates $\gamma$ are displayed in the two upper panels, and reconnecting layer widths $\delta$ are displayed in the two lower panels. Labels $L D$ and $S D$ correspond to the large- $\Delta^{\prime}$ and small- $\Delta^{\prime}$ limits, respectively.

$$
\gamma_{S D} \sim k\left(\Delta^{\prime}\right)^{2} d_{e}^{3}, \quad \delta_{S D} \sim \Delta^{\prime} d_{e}^{2}
$$

Again, we do not present here the numerical results of this case, as they have been already addressed elsewhere, ${ }^{29,56}$ but we note that we found the condition $d_{e} \gg \rho_{s}$ delimiting the validity of (36) and (37) to be numerically satisfied already for $d_{e} / \rho_{s} \geqslant 0.1$.

In this cold, collisionless case, the fastest growing mode in a large aspect ratio current sheet has been first considered in Ref. 33, where the scaling $\gamma_{M} \sim d_{e}^{3 / 2}$ has been numerically obtained for the equilibrium $\psi_{0}=\cos (x)$. This agrees with the more general estimates given in Ref. 29,

$$
\begin{gathered}
\text { Cold - Inertial } \\
\text { Regime }\left(d_{e}^{2} \gg \rho_{s}^{2}\right) \\
\text { for } \lim _{k \rightarrow 0} \Delta^{\prime} \sim k^{-p}
\end{gathered} \quad\left\{\begin{array}{l}
\gamma_{M} \sim d_{e}^{\frac{1+p}{p}}, \\
k_{M} \sim d_{e}^{\frac{1}{p}}, \\
\delta_{M} \sim d_{e},
\end{array}\right.
$$

which have been verified also for a Harris pinch equilibrium ${ }^{29}$ and, here, for equilibrium (6) (cf. Sec. VIII).

Finally, Fig. 7 evidences the formal equivalence of $d_{e}$ and $S^{-1}$ by showing the practically identical shape of the eigenfunctions obtained when inertia and resistivity, respectively, are the only non-ideal mechanisms at play and when the values are chosen so that $\gamma d_{e}^{2}=S^{-1}$. We recall instead that $\rho_{s}$, like $R^{-1}$, is per se not sufficient to regularize the singularity that, in ideal MHD, would develop at the neutral line since $\rho_{s}\left(\right.$ or $\left.R^{-1}\right)$ does not violate the topological invariance of magnetic field lines in ideal MHD, as they are dragged by the flow: although, as $d_{e}=S^{-1}=0, \rho_{s}$ appears in front of a source term in the equation for the Lagrangian invariance of $\psi$, it can be re-absorbed in a redefinition of the fluid stream function with respect to which the total (i.e., material) time derivative of $\psi$ results to be zero $\left(\varphi \rightarrow \varphi-\rho_{s}^{2} U\right)$. More in general, both $\rho_{s}$-corrections to the $\boldsymbol{E} \times \boldsymbol{B}$ velocity, as they are derived in the $\delta B_{z} \sim\left(\left|\boldsymbol{B}_{\text {perp }}\right| / B_{0}\right)^{2}$ limit, and the $\boldsymbol{J} \times \boldsymbol{B}$ term in Ohm's law of Hall-MHD model do not allow, alone, reconnection (see, e.g., Ref. 57). After redefinition of the fluid velocity, both terms can be indeed re-absorbed into a null Lie-derivative of $\boldsymbol{B}(\boldsymbol{x}, t)$, which expresses the impediment of magnetic reconnection.?

\section{INERTIAL-RESISTIVE REGIME $\left(d_{e}, S^{-1}\right)$}

In this regime, where $R^{-1}=\rho_{s}=0$, the coefficients of Eq. 5 become $\mathscr{A}=\frac{\partial^{2}}{\partial x^{2}}-k^{2}, \quad \mathscr{D}=1+k^{2} d_{e}^{2}-d_{e}^{2} \frac{\partial^{2}}{\partial x^{2}}, \quad \mathscr{L}_{1}=S^{-1} \mathscr{A}$, $\mathscr{L}_{2}=-k F_{0}^{\prime}, \mathscr{L}_{3}=k \psi_{0}^{\prime} \mathscr{A}-i k \psi_{0}^{\prime \prime \prime}$, and $\mathscr{L}_{4}=0$.

Three regions in parameter spaces can be identified, depending on the relative magnitude of $\gamma d_{e}^{2}$ and $S^{-1}$. These three regions can be classified as two asymptotic regimes, in which resistivity or electron inertia, respectively, dominate, and an intermediate region at the intersection of these two regimes.

The existence of these three regions, and in particular of a parameter range in which both $d_{e}$ and $S^{-1}$ contribute to the growth rate when $\gamma d_{e}^{2} \sim S^{-1}$, had been already noted in the pioneering numerical study of Ref. 33 although specific scalings have been there provided for the $\gamma d_{e}^{2} \gg S^{-1}$ limit only.

The scalings in the two asymptotic regimes are easily obtained.

The first regime, valid for $\gamma d_{e}^{2} \ll S^{-1}$, can be so identified as purely resistive. This has been already discussed and summarized with Eqs. (17), (18), and (23): in this case, the inertia-related terms in Eq. (1) can be neglected with respect to the resistivity contribution.

The second one, valid in the opposite limit $\gamma d_{e}^{2} \gg S^{-1}$, is the cold-inertial regime of Sec. VII. 

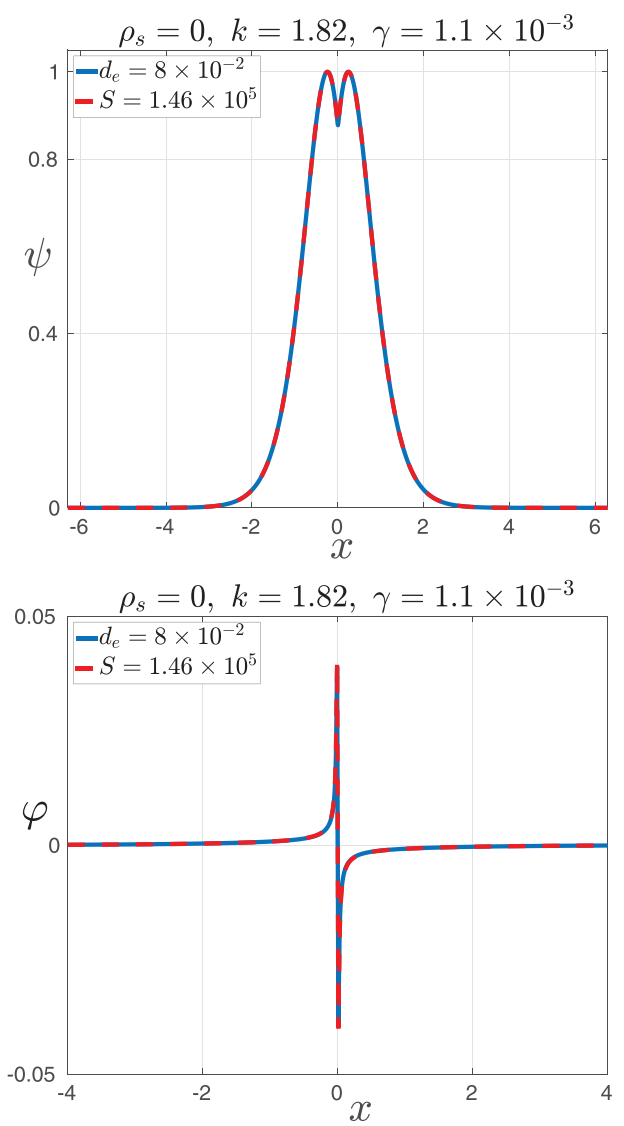

FIG. 7. Eigenmode profiles of $\psi$ (upper panel) and $\varphi$ (lower panel) obtained for a case in which $d_{e}$ (blue curve) and $S^{-1}$ (red curve) are the only non-zero non-ideal parameters. The values of the parameters have been chosen so that $\gamma d_{e}^{2} \simeq S^{-1}$; the blue and red curves are practically superimposable.

The intermediate parameter range is defined by the constraint $\gamma d_{e}^{2} \sim S^{-1}$. We note that the scalings for tearing modes in the presence of both electron inertia and resistivity can be deduced by considering, for example, the scalings (36) and (37) of the cold inertial case and by rescaling ${ }^{56} d_{e}^{2} \rightarrow \hat{d}_{e}{ }^{2} \equiv d_{e}^{2}+S^{-1} / \gamma$. For the growth rates, this leads to the implicit relations

$$
\gamma_{L D} \sim k\left(d_{e}^{2}+\frac{S^{-1}}{\gamma_{L D}}\right)^{1 / 2}, \quad \gamma_{S D} \sim k\left(\Delta^{\prime}\right)^{2}\left(d_{e}^{2}+\frac{S^{-1}}{\gamma_{S D}}\right)^{3 / 2},
$$

which are useful to interpret the results in the transition region.

Using for $\gamma$ the scalings [(36) and (37)] of the collisionless tearing modes or the scalings [(17) and (18)] of the purely resistive case, it is possible to reformulate the condition of transition between the two asymptotic regimes in terms of the wave-vector as

$$
\text { Inertial - resistive }\left\{\begin{array}{l}
k \sim d_{e}^{-3} S^{-1} \quad\left(\Delta^{\prime} \delta \gg 1\right), \\
\text { range } \gamma d_{e}^{2} \sim S^{-1} \\
k\left(\Delta^{\prime}\right)^{2} \sim d_{e}^{-5} S^{-1} \quad\left(\Delta^{\prime} \delta \ll 1\right) .
\end{array}\right.
$$

In a numerical scan of the parameter space, it is easier to identify the three regions (i.e., the two asymptotic regimes and the transition region) by focusing first on the scalings of the fastest growing mode, for which the wave-vector is fixed by the non-ideal parameters.

Figure 8 displays the results of a set of numerical integrations performed by varying both $d_{e}$ and $S^{-1}$ and by selecting the corresponding fastest growing mode in a formally continuous spectrum of unstable modes. The dependence of $\gamma_{M}$ and $k_{M}$ on $d_{e}$ and on $S^{-1}$ is displayed in the top and bottom frames, respectively. For each line, the transition from one asymptotic regime to the other, occurring when $\gamma d_{e}^{2} \sim S^{-1}$, is quite evident. In each frame, the dashed line evidences the scaling dependence on the dominant parameter of the mostly inertial (38) or mostly resistive regime (25).

\section{DISCUSSION}

The main results presented in Sec. V-VII are summarized in Table I, where we have reported only the scalings for the regimes where both non-ideal parameters each time involved contribute. We recall that only the scalings of the fastest growing mode depend explicitly on the analytical form of the perturbed magnetic equilibrium but not those of the small- and large- $\Delta^{\prime}$ limits. For the scalings of the fastest growing mode, we have indicated also the values that can be extrapolated for an equilibrium for which $p=1$ when $\Delta^{\prime}(k) \sim k^{-p}$ for $k \ll 1$, according to the criterion discussed in Ref. 29 and previously recalled; in the present numerical study, we have verified only the case $p=2$ of equilibrium (6). Different types of equilibria and their corresponding algebraic scaling in terms of $k$ for $k \ll 1$ are reported in Table II.

While the scalings of the viscous-resistive regime had been already analytically obtained and numerically verified by previous authors, also for the fastest growing mode, a different choice of the magnetic equilibrium had been made, which corresponds to the $p=1$ case (cf. Sec. V). The scalings of the warm-resistive regimes were instead available only for the growth rates and in the small- and large$\Delta^{\prime}$ limits (Sec. VI). In the inertial-resistive regime (Sec. VIII), for which no power law scaling can be measured in the narrow parameter region in which both resistivity and electron inertia contribute, a numerical scan spanning the two asymptotic regimes at the best of our knowledge had never been performed before.

The warm resistive scaling, once combined with those of the warm-inertial regime in the tearing and inertial-kink limits and for the fastest growing mode (see Sec. VI-VII), can provide, for example, a correction to the estimate previously done in Ref. 59, about the possible onset of a secondary inertial tearing on the current sheet generated by a primary resistive internal kink mode (for example, in a tokamak, during the sawtooth crash in Kadomtsev scenario). In this case, the embedded current sheet condition ${ }^{60}$ must be kept into account to evaluate the scalings (see also Ref. 61).

Moreover, while in the present work we have carried out the linear analysis assuming a static equilibrium, the question of how a thin current sheet of a given aspect ratio $L / a$ is produced is pertinent to assess what happens in a given context.

If one assumes that the current sheet is generated by an underlying dynamical process whose characteristic timescale is $\tau_{\text {proc }}$, it is important to compare this timescale with the growth time of all the unstable modes permitted at a given sheet aspect ratio.

If the process timescale is sufficiently long, one expects that the current sheet bifurcates to a state with a magnetic island determined by the characteristics of the first mode that becomes unstable. On the 

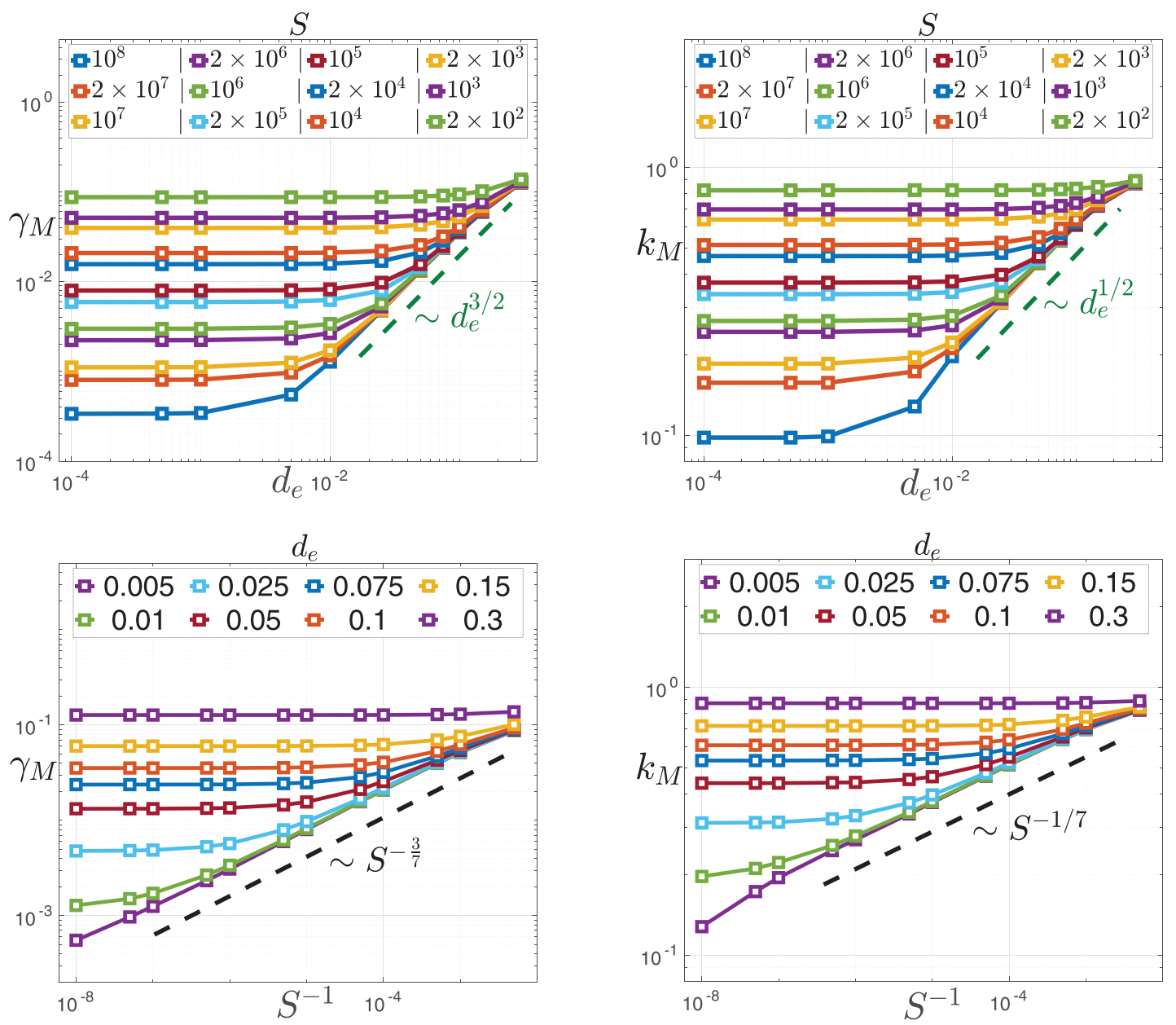

FIG. 8. Scaling laws of the fastest growing mode occurring for $\Delta^{\prime} \delta \sim 1$ in the inertial-resistive regime. The dashed green lines of the upper frames indicate the scaling of the purely collisionless regime (38), while the black ones in the lower frames indicate the scalings of the purely resistive regime (25).

other hand, if the underlying process is fast enough that $\tau_{\text {proc }}$ is smaller than the growth times of all the unstable modes permitted at a given aspect ratio, then one expects that the process of producing thinner and thinner sheets can go on until this condition is violated. An example occurs when $\tau_{\text {proc }}$ is Alfvénic, as discussed in the example below, for a mechanism leading to thin sheets exhibiting both ranges of the unstable spectrum.

\section{A. A case of current sheet evolution}

Consider a (typically turbulent) plasma characterized by eddies of size $L$, fluid velocity $v_{f}$ and magnetic field $B_{0}$. Assume energy equipartition, such that $v_{f} \sim v_{A}$, where $v_{A}$ is the Alfvén velocity associated with the field $B_{0}$. One can, then, consider a process by which current sheets are produced as the magnetic field is advected by the plasma motion on a timescale $\tau_{\text {proc }}$ of the order of the eddy turnover time $\tau_{f} \sim L / v_{f}$, which is also of the order of the Alfvén time $\tilde{\tau}_{A} \sim L / v_{A}$ (the symbol " $\tilde{\tau}_{A}$," here, is to mark the difference with respect to $\tau_{A}=a / v_{A}$ previously introduced).
Assume also that the current $J_{0}$ in the sheet scales like $B_{0} / a$ (i.e., $\left.B_{c s} \sim B_{0}\right)$. One has, therefore, to take into account that, as the sheet shrinks, $a$ gets smaller and $\tau_{0}$, which in this specific case can be written as $\tau_{0} \sim(a / L) \tilde{\tau}_{A}$, also gets smaller.

Of course, the linear analysis on a static equilibrium would be applicable only if the unstable modes satisfied $\gamma \tau_{\text {proc }} \gg 1$, which here means $\gamma \tau_{0} \sim \gamma \tau_{A} \gg 1$. However, since only modes with $\gamma \tau_{0} \ll 1$ can be destabilized if the aspect ratio is not large enough, a critical condition of transition corresponding to $\gamma \tau_{0} \sim 1$ can be determined by performing a stability analysis on a sequence of static current sheets ordered according to the increasing values of $L / a$. It is in the sense that below we mean the application of the linear estimates to the rapidly evolving current sheet.

As the inverse aspect ratio grows, more and more modes become unstable, initially in the small $\Delta^{\prime}$ range. Of these, the fastest growth is associated with the smallest possible wave-number, which is $k \sim 1 / L$. This process goes on until the condition $\Delta^{\prime} \delta \approx 1$ is met for this mode.

At this point, it is necessary to make an assumption about the dependence of $\Delta^{\prime}$ on $k$ and $a$. Let us assume, for example, 
TABLE I. Asymptotic scalings of resistive reduced-MHD tearing modes in the presence of viscosity and electron temperature and of collisionless modes in the presence of electron temperature. The bottom line refers to the scaling of the critical aspect ratio $(a / L)_{c r i t}$ discussed in Sec. IXA.

\begin{tabular}{|c|c|c|c|c|c|c|}
\hline & Viscous-resisti & $($ Sec. V) & Warm-resisti & (Sec. VI) & Warm-inertial: $\left(\rho_{s}, d_{e}\right)$ & (Sec. VII) \\
\hline$\gamma_{L D}$ & \multicolumn{2}{|c|}{$k^{\frac{2}{3}} R^{\frac{1}{3}} S^{-\frac{2}{3}}$} & \multicolumn{2}{|c|}{$k^{\frac{6}{7}} \rho_{s}^{\frac{4}{7}} S^{-\frac{1}{7}}$} & \multicolumn{2}{|l|}{$k \rho_{s}^{\frac{2}{3}} d_{e}^{\frac{1}{3}}$} \\
\hline$\delta_{L D}$ & \multicolumn{2}{|c|}{$k^{-\frac{1}{3}} R^{-\frac{1}{6}} S^{-\frac{1}{6}}$} & \multicolumn{2}{|c|}{$k^{-\frac{4}{7}} \rho_{s}^{-\frac{5}{7}} S^{-\frac{4}{7}}$} & \multicolumn{2}{|l|}{$\rho_{s}^{-\frac{1}{3}} d_{e}^{\frac{4}{3}}$} \\
\hline$\gamma_{S D}$ & \multicolumn{2}{|c|}{$k^{\frac{1}{3}} \Delta^{\prime} R^{\frac{1}{6}} S^{-\frac{5}{6}}$} & \multicolumn{2}{|c|}{$\left(k \Delta^{\prime}\right)^{\frac{2}{3}} \rho_{S}^{\frac{2}{3}} S^{-\frac{1}{3}}$} & \multicolumn{2}{|l|}{$k \Delta^{\prime} \rho_{s} d_{e}$} \\
\hline$\delta_{S D}$ & \multicolumn{2}{|c|}{$k^{-\frac{1}{3}} R^{-\frac{1}{6}} S^{-\frac{1}{6}}$} & \multicolumn{2}{|c|}{$k^{-\frac{2}{3}} \Delta^{J^{\frac{1}{3}}} \rho_{s}^{-\frac{2}{3}} S^{-\frac{2}{3}}$} & \multicolumn{2}{|l|}{$\Delta^{\prime} d_{e}^{2}$} \\
\hline & $\Delta^{\prime} \sim(k a)^{-1}$ & $\Delta^{\prime} \sim(k a)^{-2}$ & $\Delta^{\prime} \sim(k a)^{-1}$ & $\Delta^{\prime} \sim(k a)^{-2}$ & $\Delta^{\prime} \sim(k a)^{-1}$ & $\Delta^{\prime} \sim(k a)^{-2}$ \\
\hline$\gamma_{M}$ & $R^{\frac{1}{4}} S^{-\frac{3}{4}}$ & $R^{\frac{2}{7}} S^{-\frac{5}{7}}$ & $\rho_{S}^{\frac{2}{3}} S^{-\frac{1}{3}}$ & $\rho_{S}^{\frac{5}{8}} S^{-\frac{1}{4}}$ & $\rho_{s} d_{e}$ & $\rho_{s}^{\frac{5}{6}} d_{e}^{\frac{2}{3}}$ \\
\hline$k_{M}$ & $\left(R^{-\frac{1}{2}} S^{-\frac{1}{2}}\right)^{\frac{1}{4}}$ & $\left(R^{-\frac{1}{2}} S^{-\frac{1}{2}}\right)^{\frac{1}{7}}$ & $\rho_{s}^{\frac{1}{9}} S^{-\frac{2}{9}}$ & $\rho_{s}^{\frac{1}{16}} S^{-\frac{1}{8}}$ & $\rho_{s}^{\frac{1}{3}} d_{e}^{\frac{2}{3}}$ & $\rho_{s}^{\frac{1}{6}} d_{e}^{\frac{1}{3}}$ \\
\hline$\delta_{M}$ & $\left(R^{-\frac{1}{2}} S^{-\frac{1}{2}}\right)^{\frac{1}{4}}$ & $\left(R^{-\frac{1}{2}} S^{-\frac{1}{2}}\right)^{\frac{2}{7}}$ & $\rho_{s}^{-\frac{7}{9}} S^{-\frac{4}{9}}$ & $\rho_{s}^{-\frac{3}{4}} S^{-\frac{1}{2}}$ & $\rho_{s}^{-\frac{1}{3}} d_{e}^{\frac{4}{3}}$ & $\rho_{s}^{-\frac{1}{3}} d_{e}^{\frac{4}{3}}$ \\
\hline$\left(\frac{a}{L}\right)_{\text {crit }}$ & $R^{* \frac{1}{6}} S^{*-\frac{1}{2}}$ & $R^{* \frac{1}{10}} S^{*-\frac{1}{2}}$ & $\rho_{s}^{* \frac{1}{3}} S^{*-\frac{1}{6}}$ & $\rho_{s}^{* \frac{1}{3}} S^{*-\frac{2}{15}}$ & $\rho_{s}^{* \frac{1}{3}} d_{e}^{\frac{* 1}{3}}$ & $\rho_{s}^{* \frac{1}{3}} d_{e}^{* \frac{4}{15}}$ \\
\hline
\end{tabular}

the scaling $\Delta^{\prime} \sim\left(k a^{2}\right)^{-1}$, which is what one gets for the Harris pinch at small $k$ [the argument can be generalized to different power-law scalings, ${ }^{29}$ among which equilibrium (6) - see next and Table II, at the end].

One finds eventually that the maximally growing mode appears in the spectrum at $k \sim 1 / L$ when $a / L \sim S_{L}^{-1 / 5}$, where $S_{L}=L^{2} /$ $\left(\eta_{m} \tilde{\tau}_{A}\right)$ is the Lundquist number evaluated with the large scale parameters. The corresponding layer width is $\delta \sim L S_{L}^{-2 / 5}$, and the maximum growth rate is such that

$$
\gamma \tilde{\tau}_{A} \sim S_{L}^{-1 / 5} .
$$

We note that the growth rate is still smaller than the characteristic rate at which the current sheet shrinks (by assumption, $\sim \tilde{\tau}_{A}^{-1}$ ). Therefore, the shrinking process can continue producing modes in the lower wave-number portion of the spectrum, which is now in the large $\Delta^{\prime}$ range. "Producing" is here meant in the sense that the aspect ratio grows up to values, which, in a static current sheet, would allow the destabilization of large $\Delta^{\prime}$ modes.
One can, thus, evaluate that the wavenumber $k_{M}$ of the fastest growing mode scales now as

$$
k_{M} L \sim S_{L}^{-1 / 4}\left(\frac{L}{a}\right)^{5 / 4},
$$

and the maximum growth rate $\gamma_{M}$ scales as

$$
\gamma_{M} \tilde{\tau}_{A} \sim S_{L}^{-1 / 2}\left(\frac{L}{a}\right)^{3 / 2} .
$$

The critical aspect ratio, when $\gamma_{M} \sim 1 / \tilde{\tau}_{A}$, is, therefore, reached when

$$
\left(\frac{L}{a}\right)_{\text {crit }} \sim S_{L}^{1 / 3} .
$$

This is the smallest possible aspect ratio compatible with the stability condition. Below this value, the current sheet would be destroyed by a mode growing at a faster rate than the one at which the sheet is produced.

TABLE II. Equilibrium profiles and related expression of $\Delta^{\prime}(k a)$ (both with a bibliographical reference about where the former had been first used for tearing mode analysis and about where a formula for the second has been provided) and the corresponding scaling of $\Delta^{\prime}(k a)$ with respect to $k a$ for $k a \ll 1$. In this table, dimensional units have been reintroduced.

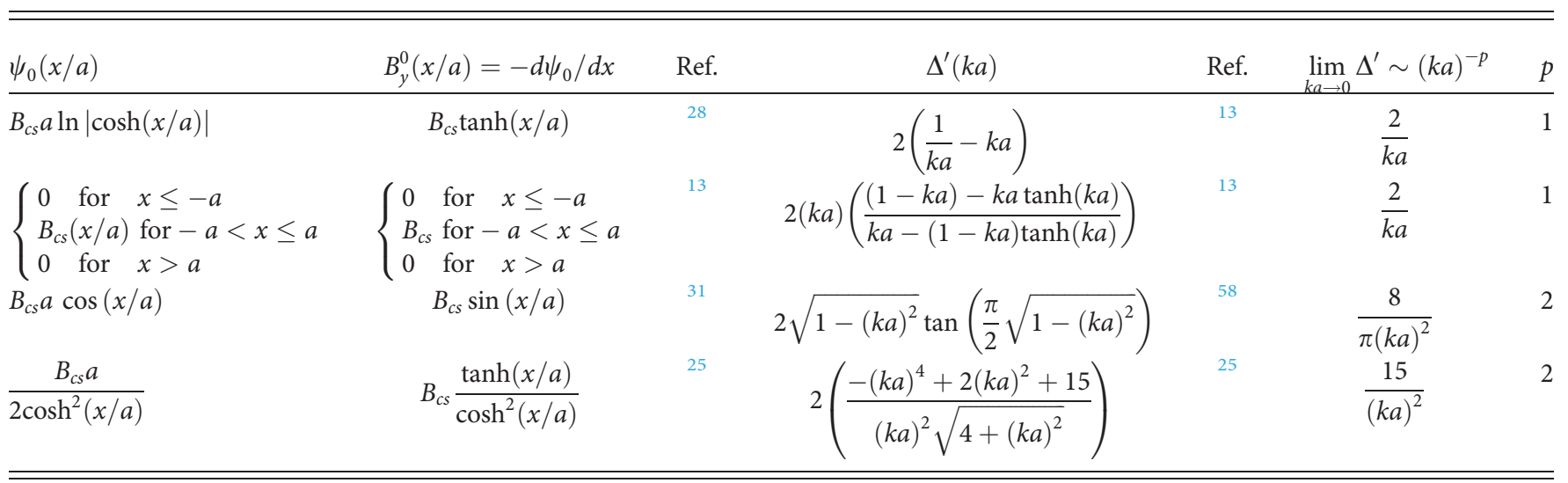


The corresponding critical wave-number scales as

$$
\left(k_{M} L\right)_{\text {crit }} \sim S_{L}^{1 / 6}
$$

which gives a measure of the size of the smaller structures produced when the sheet is destroyed, a quantity that can, in principle, be compared with observations.

Although in the different context of the forced evolution of a single current sheet shrinking at an Alfvénic rate, conditions (44) and (45) had been already proven numerically by Tenerani et al. ${ }^{62}$ to provide the threshold condition for the disruption of the evolving current sheet. The results (44) and (45) are indeed formally coincident with the scalings of the ideal tearing regime, ${ }^{49}$ introduced by Pucci and Velli in terms of an asymptotic threshold condition on the aspect ratio of a microscopically thin current sheet, at which a resistive tearing would be allowed to develop over Alfvénic time scales: for larger asymptotic scalings, super-Alfvénic modes ${ }^{63-65}$ with $\gamma_{M} \tilde{\tau}_{A} \stackrel{\eta_{m} \rightarrow \infty}{\longrightarrow} \infty$ would be, in principle, admitted.

The choice of Velli and coworkers for this name was motivated by the independence from $S_{L}$ of the growth rate $\gamma_{I T}$, which is numerically estimated as the limit toward which $\gamma_{M}\left(S_{L}^{-1}\right)$ converges, while the critical aspect ratio condition (44) for $(L / a)_{\text {crit }}$ is imposed to be satisfied as increasingly smaller values of $S_{L}^{-1}$ are chosen. Although this notion of "ideal tearing" has been shown in Ref. 29 to go in principle beyond the Alfvénic case and to be generalizable to any reconnection regime (i.e., regardless of the nonideal effect at play and of the "reference" time scales that can be non-Alfvénic), here we want to stress that the critical conditions of Eqs. (44) and (45) have been obtained in the well precise context of a current sheet formation mechanism and that is the latter that defines and fixes the "timescale of reference," $\tau_{\text {proc }}$, of the system. In principle, $\tau_{\text {proc }}$ does not coincide with $\tau_{A}$ (or with the characteristic inverse frequency of the linear stable modes of the system). It is also important to keep in mind that the result is dependent on specific assumptions about the dependence of the current density and of $\Delta^{\prime}$, on the width of the sheet, and on the wavenumber (see also Ref. 61 for further discussions).

\section{B. Critical scalings in the viscous-resistive, warm-resistive, and warm-inertial regimes}

The critical scalings [(44) and (45)] can be of course generalized to any reconnection regime, similarly to what was shown ${ }^{29}$ to be possible for the ideal tearing case. Let us do this in the regimes discussed in Sec. V-VII. From a practical point of view, it is sufficient to consider the scalings of the fastest growing modes that we have provided as written by normalizing lengths to $a$ and magnetic fields to $B_{0}$ and to rewrite them as normalized to $L$. Let us then label with an apex “*” the dimensionless quantities referred in this way to $L$. For the kind of problem discussed above, $\tilde{\tau}_{A}=\tau_{A}^{*}$ is, thus, the characteristic time of the process governing the current sheet evolution and the limit $\gamma_{M}^{*} \sim O(1)$ must be posed for consistency with the linear analysis. This provides an asymptotic scaling of the critical aspect ratio $(L / a)_{\text {crit }}$ with the non-ideal parameters involved.

In the viscous-resistive regime of Sec. V, we, thus, rewrite Eq. (22) as

$$
\left\{\begin{array}{l}
\gamma_{M}^{*} \sim\left(\frac{L}{a}\right)^{\frac{2+4 p}{1+3 p}} R^{* \frac{p}{1+3 p}} S^{*-\frac{1+2 p}{1+3 p}}, \\
k_{M}^{*} \sim\left(\frac{L}{a}\right)^{\frac{2+3 p}{1+3 p}}\left(R^{*-1 / 2} S^{*-1 / 2}\right)^{\frac{1}{1+3 p}}, \\
\delta_{M}^{*} \sim\left(\frac{L}{a}\right)^{-\frac{1+2 p}{1+3 p}}\left(R^{*-1 / 2} S^{*-1 / 2}\right)^{\frac{p}{1+3 p}} .
\end{array}\right.
$$

The critical scalings (44) and (45) so read

$$
\begin{gathered}
\left(\frac{L}{a}\right)_{\text {crit }} \sim R^{*-\frac{p}{2+4 p}} S^{* \frac{1}{2}}=P^{*-\frac{p}{2+4 p}} S^{* \frac{1+p}{2+4 p}}, \\
\left(k_{M} L\right)_{\text {crit }} \sim R^{*-\frac{1+p}{2+4 p}} S^{* 1 / 2} .
\end{gathered}
$$

This result can be specialized: for $p=1$, it corresponds to both the Harris-pinch equilibrium $\psi_{0}=a \ln |\cosh (x / a)|$ and the piece-wise equilibrium defined by $\psi_{0}(x)=x / a$ in the interval $-a<x \leq a$ and by $\psi_{0}=0$ for $x \leq-a$ and $x>a$ (Ref. 13). For $p=2$, it corresponds to the case of Eq. (6) and the case of the equilibrium $\psi_{0}(x)=a \sin (x / a)$ (Ref. 58),

$$
\left(\frac{L}{a}\right)_{\text {crit }}^{[p=1]} \sim \sim R^{*-\frac{1}{6}} S^{* \frac{1}{2}}, \quad\left(\frac{L}{a}\right)_{\text {crit }}^{[p=2]} \sim \sim R^{*-\frac{1}{10}} S^{* \frac{1}{2}}
$$

The scalings of $\gamma_{M}^{*}$ in Eq. (46) and the critical condition of Eqs. (47) -(49) specialized to the $p=1$ case had been first obtained numerically by Tenerani et al. in Ref. 2 .

A current sheet with an aspect ratio (asymptotically) larger than that indicated above is met, for example, in the case of a Sweet-Parker type steady-reconnection assumption on the current sheet aspect ratio. In the purely resistive case, it corresponds to $L / a \sim S_{L}^{1 / 2}$. Its application to the evaluation of the fastest growing mode in the purely resistive regime gives ${ }^{63,64} \gamma_{M}^{*} \sim S_{L}^{1 / 4}$ : in the literature, this has been often referenced to as the plasmoid instability regime. ${ }^{64,65}$ In the viscousresistive case, the corresponding assumption has been discussed in Ref. 66 (see also Ref. 67).

Analogous results are obtained in the warm-resistive regime of Sec. VI. Equations (32), re-scaled to $L$, become

$$
\left\{\begin{array}{l}
\gamma_{M}^{*} \sim\left(\frac{L}{a}\right)^{\frac{6+12 p}{2+7 p}} \rho_{s}^{* \frac{2+4 p}{2+7 p}} S^{*-\frac{2+p}{2+7 p}}, \\
k_{M}^{*} \sim\left(\frac{L}{a}\right)^{\frac{5+7 p}{2+7 p}} \rho_{s}^{* \frac{1}{2+7 p}} S^{*-\frac{2}{2+7 p}}, \\
\delta_{M}^{*} \sim\left(\frac{L}{a}\right)^{-\frac{4+8 p}{2+7 p}} \rho_{s}^{*-\frac{2+5 p}{2+7 p}} S^{*-\frac{4 p}{2+7 p}} .
\end{array}\right.
$$

Here, the critical aspect ratio and fastest growing mode read

$$
\begin{gathered}
\left(\frac{L}{a}\right)_{\text {crit }} \sim \rho_{s}^{*-\frac{1}{3}} S^{* \frac{2+p}{3(2+4 p)}}, \\
\left(k_{M} L\right)_{\text {crit }} \sim \rho_{s}^{*-\frac{1}{3}} S^{* \frac{p-1}{2(3+4 p)}} .
\end{gathered}
$$

As it happens in the warm-collisionless case, ${ }^{29}$ the equilibrium choice does not affect the scaling dependence of the critical aspect ratio on $\rho_{s}$. For $p=1$ and $p=2$, Eq. (51) specializes to 


$$
\left(\frac{L}{a}\right)_{\text {crit }}^{[p=1]} \sim \rho_{s}^{*-\frac{1}{3}} S^{* \frac{1}{6}}, \quad\left(\frac{L}{a}\right)_{\text {crit }}^{[p=2]} \sim \rho_{s}^{*-\frac{1}{3}} S^{* \frac{2}{15}} .
$$

Also note that for $p=1$, the critical wave vector does not depend on resistivity.

For completeness we finally write the critical scalings already evaluated in Ref. 29 for the warm-inertial regime,

$$
\left\{\begin{array}{l}
\gamma_{M}^{*} \sim\left(\frac{L}{a}\right)^{\frac{1+2 p}{p}} \rho_{s}^{* \frac{1+2 p}{3 p}} d_{e}^{* \frac{2+p}{3 p}} \\
k_{M}^{*} \sim\left(\frac{L}{a}\right)^{\frac{1+p}{p}} \rho_{s}^{* \frac{1}{3 p}} d_{e}^{* \frac{2}{3 p}} \\
\delta_{M}^{*} \sim \rho_{s}^{*-\frac{1}{3}} d_{e}^{* \frac{4}{3}}
\end{array}\right.
$$

The critical aspect ratio and fastest growing mode are

$$
\begin{aligned}
& \left(\frac{L}{a}\right)_{\text {crit }} \sim \rho_{s}^{*-\frac{1}{3}} d_{e}^{*-\frac{2+p}{3(1+2 p)}}, \\
& \left(k_{M} L\right)_{\text {crit }} \sim \rho_{s}^{*-\frac{1}{3}} d_{e}^{*-\frac{p-1}{3+4 p}},
\end{aligned}
$$

and for $p=1$ and $p=2$, the critical radii read

$$
\left(\frac{L}{a}\right)_{\text {crit }}^{[p=1]} \sim \rho_{s}^{*-\frac{1}{3}} d_{e}^{*-\frac{1}{3}}, \quad\left(\frac{L}{a}\right)_{\text {crit }}^{[p=2]} \sim \rho_{s}^{*-\frac{1}{3}} d_{e}^{*-\frac{4}{15}} .
$$

These results perfectly mirror those of the warm-resistive case according to the correspondence $S^{-1} \leftrightarrow \gamma d_{e}^{2}$, which here reads $S^{-1} \leftrightarrow d_{e}^{2}$ since the critical radii above are the same as of the ideal tearing, $\gamma_{M}^{*}=\gamma_{I T} \sim O(1)$.

The critical scalings we have written in the three regimes are summarized in the last line of Table I.

It should be noted that it is left open the question of whether values of $p$ different from 1 and 2 are admitted for different magnetic equilibrium profiles with a single resonant point. The change in the regime indicated by Eqs. (52) and (56) when $p<1$ suggests us to exclude the possibility of fractional values smaller than unity.

\section{SUMMARY AND CONCLUSIONS}

We have presented and discussed numerical results about the scalings of tearing modes in a slab geometry for different couples of non-ideal parameters, simultaneously contributing to RMHD equations. The wavelength limits corresponding to the small- $\Delta^{\prime}$, fastest growing mode and large- $\Delta^{\prime}$ limits have been all addressed. Although the scalings of all growth rates in the small- and large- $\Delta^{\prime}$ limits were already available from boundary layer analysis, to the best of our knowledge, such estimates had not been numerically tested, before, in all the regimes considered here. The present study contributes to the subject by complementing a systematic numerical verification with the scalings of the reconnecting layer width, $\delta$, not always available in the pre-existing literature and by providing the scaling of the fastest growing modes for a particular choice of the magnetic equilibrium profile. In most reconnection regimes, the latter were not previously available or had been provided for different magnetic equilibrium choices. Comparison of all results confirms the general heuristic estimates proposed in a previous work, which we have here re-considered and extended to all the regimes addressed. Implications of this analysis have been, then, discussed for an example of current sheet whose aspect ratio evolves over an Alfvénic timescale: critical scalings of the smallest observable aspect ratio have been so obtained, which depend on the equilibrium profile and, in each reconnection regime, are formally coincident with those of the geometrical threshold for the transition to the recently proposed ideal tearing ${ }^{49}$ asymptotic limit. Finally, while discussing numerical details of the finite-difference eigensolver that we have developed on purpose, we have addressed the role played by ill-conditioning in seeking a compromise between the convergence of the solver, the accuracy of the stencil, the non-uniformity of the numerical grid, and machine precision.

It is our hope that the organic presentation of the scalings here discussed may find use in a broad range of applications, ranging from the linear benchmarks of numerical codes to the interpretation of numerical and experimental results in tokamak physics and in studies of turbulent reconnection, both in the slab RMHD limit with no Hall term and in the low- $\beta$ reduced Hall-MHD regime. Moreover, this work represents a first step toward a systematic multiparametric numerical analysis of tearing modes in which more than two nonideal parameters simultaneously contribute.

\section{ACKNOWLEDGMENTS}

We thank Erwan Deriaz (CNRS) for having suggested us the general form of the non-uniform grid profile Eq. (B1) and for useful discussions about numerical convergence issues and Francesco Pegoraro (Università di Pisa) for insightful comments on the original manuscript. We also wish to thank the "Maison de la simulation Lorraine" for partial time allocation on the cluster Explor (Project No. 2019M4XXX0978). This work was carried out within the framework of the French Federation for Magnetic Fusion Studies (FR-FCM) and of the Eurofusion consortium and received funding from the Euratom research and training programme 2014-2018 and 2019-2020 under grant agreement No. 633053 (WPEDU fundings obtained through FR-FCM AAP 2017-2019 "Evolution of current sheets in low-collision plasmas," in particular, are gratefully acknowledged). The views and opinions expressed herein do not necessarily reflect those of the European Commission.

\section{APPENDIX A: A TTH-ORDER ACCURATE FINITE DIFFERENCE STENCIL FOR THE NTH DERIVATIVE ON A NON-UNIFORM GRID}

In the eigenesolver, derivatives are numerically computed using the standard compact difference method. ${ }^{68}$ Here, we present a general, explicit form for a non-uniform approximation of the $n$th derivative on a non-uniform grid by using a centered stencil with $N_{t}$ points and accuracy up to order $t=N_{t}-n$. It reads

$$
\left.\frac{\partial^{n} f}{\partial x^{n}}\right|_{i}=\sum_{m=-\frac{N_{t}-1}{2}}^{\frac{N_{t}-1}{2}} a_{i+m} f_{i+m}+e^{\left(t^{t h}\right)}
$$

Note that, even though the stencils we are considering are centered (and therefore, $N_{t}$ is an odd number), they are, in general, not symmetric because of the non-uniformity of the grid (see Appendix B). 
Coefficients $a_{i+m}$ are evaluated by following the standard compact difference approach, using Taylor expansion. For the points to the left and to the right of the center of the stencil, $x_{i}$, we find, respectively,

$$
\begin{gathered}
f_{i-j}=\left.\sum_{m=0}^{N_{t}-1} \frac{h_{i-j}^{l}}{m !} \frac{\partial^{m} f}{\partial x^{m}}\right|_{i}-\left.\frac{h_{i-j}^{N_{t}}}{N_{t} !} \frac{\partial^{N_{t}}}{\partial x^{N_{t}}} f\right|_{i} \\
f_{i+j}=\left.\sum_{m=0}^{N_{p}-1}(-1)^{m} \frac{h_{i+j}^{m}}{m !} \frac{\partial^{m} f}{\partial x^{m}}\right|_{i}+\left.\frac{h_{i+j}^{N_{p}}}{N_{p} !} \frac{\partial^{N_{p}}}{\partial x^{N_{p}}} f\right|_{i}
\end{gathered}
$$

Using Eqs. (A2) and (A3) combined with (A1), we obtain a system of $N_{t}$ equations for the coefficients $a_{i \pm m}$, with $1 \leq m \leq\left(N_{t}-1\right) / 2$. We write it as

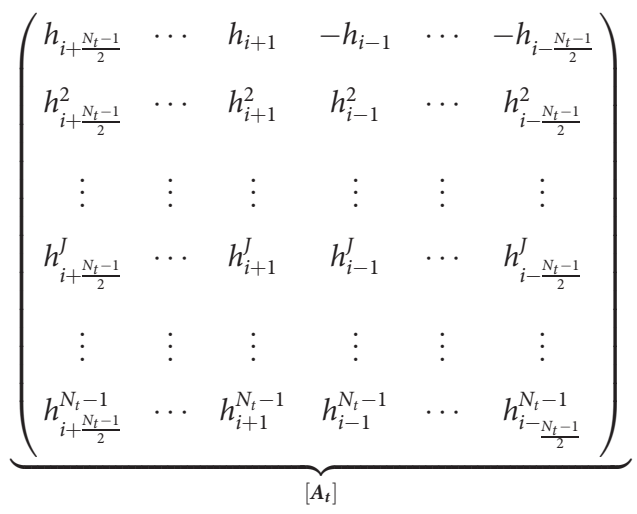

$$
\begin{aligned}
& \times \underbrace{\left(\begin{array}{c}
a_{i+\frac{N_{t}-1}{2}} \\
a_{i+\frac{N_{t}-1}{2}-1} \\
\cdot \\
\cdot \\
a_{i+\frac{N_{t}-1}{2}-J-1} \\
\cdot \\
\cdot \\
a_{i-\frac{N_{t}-1}{2}}
\end{array}\right)}_{a}=\underbrace{\left(\begin{array}{c}
0 \\
0 \\
0 \\
0 \\
J ! \\
0 \\
0
\end{array}\right)}_{\boldsymbol{b}}
\end{aligned}
$$

where " $\times$ " stands here for the matrix product. Naming $\left[\tilde{\boldsymbol{A}}_{j}\right]$ the matrix that results from replacing the column $j$ in $\left[\boldsymbol{A}_{t}\right]$ with vector $\boldsymbol{b}$, the coefficients $a_{i \pm j}$ can be found, for example, by using Cramer's method as follows:

$$
a_{i \pm j}=\frac{\operatorname{det}\left[\tilde{\boldsymbol{A}}_{j}\right]}{\operatorname{det}\left[\boldsymbol{A}_{t}\right]} \quad a_{0}=-\sum_{j=-\frac{N_{t}-1}{2}, j \neq 0}^{\frac{N_{t}-1}{2}} a_{i+j} .
$$

Above, the index $j$ runs over $j=1,2, . .,\left(N_{t}-1\right) / 2$. The determinant of $\left[\boldsymbol{A}_{\boldsymbol{t}}\right]$ can be computed using the fact that $\boldsymbol{A}_{\boldsymbol{t}}$ is a matrix of Vandermonde-type. After some algebra, we can write a general formula for calculating the determinant of a stencil matrix of size $t \times t$,

$$
\begin{aligned}
\operatorname{det}\left[\mathbf{A}_{\mathbf{t}}\right]= & \left(\prod_{l=-N_{s}, l \neq 0}^{N_{t}} h_{i+l}\right)\left(\prod_{k=1}^{N_{t}-1} \prod_{l=k+1}^{N_{t}}\left[h_{i \pm k}-h_{i \pm l}\right]\right) \\
& \times\left(\prod_{k=1}^{N_{t}} \prod_{l=1}^{N_{t}}\left[h_{i+k}+h_{i-l}\right]\right),
\end{aligned}
$$

with $\times$ now expressing the standard algebraic product. From Eqs. (A2) and (A3), we can, then, rewrite the local truncation error of the scheme of $t$ th $=\left(N_{t}-n\right)$ th order,

$$
e^{t}=\left[\sum_{j=1}^{N_{t}} h_{i+j}^{N_{t}} a_{i+j}-\sum_{j=1}^{N_{t}} h_{i-j}^{N_{t}} a_{i-j}\right] \times\left.\frac{1}{N_{t} !} \frac{\partial^{N_{t}} f}{\partial x^{N_{t}}}\right|_{i} .
$$

It should be noted that in Eq. (A7), the number of multiplications of ill-conditioned terms like $h_{i \pm k}-h_{i \pm l}$ increases with the sought accuracy $t$ of the scheme: this provides a source of rounding-error, which eventually forces to reduce the machine-epsilon (i.e., to increase the precision of number representation). Notice that this is an unavoidable drawback of finite-difference schemes, which must be "payed" in order to overcome ill-conditioning problems, when higher order stencils are used.

Previous formulas can be used to compute the coefficients of compact schemes for an arbitrary derivative with arbitrary accuracy. As an explicit example, we can consider a fourth order accurate stencil $(t=4)$ for the first derivative $(n=1)$ such that it has been used for the numerical calculations discussed in the present article.

Using these values, whence $N_{t}=t+n=5$, Eqs. (A1), (A2), and (A3) read, respectively,

$$
\begin{gathered}
\left.\frac{\partial f}{\partial x}\right|_{i}=\sum_{m=-2}^{2} a_{i+m} f_{i+m}+e^{(4)}, \\
f_{i-j}=\left.\sum_{l=0}^{4} \frac{h_{i-j}^{l}}{l !} \frac{\partial^{l} f}{\partial x^{l}}\right|_{i}-\left.\frac{h_{i-j}^{5}}{5 !} \frac{\partial^{5} f}{\partial x^{5}}\right|_{i}, \\
f_{i+j}=\left.\sum_{l=0}^{4}(-1)^{l} \frac{h_{i+j}^{l}}{l !} \frac{\partial^{l} f}{\partial x^{l}}\right|_{i}+\left.\frac{h_{i+j}^{5}}{5 !} \frac{\partial^{5} f}{\partial x^{5}}\right|_{i} .
\end{gathered}
$$

This leads to a linear system of $N_{t}=5$ equations: to the following linear system,

$$
\underbrace{\left(\begin{array}{cccc}
h_{i+2} & h_{i+1} & -h_{i-1} & -h_{i-2} \\
h_{i+2}^{2} & h_{i+1}^{2} & h_{i-1}^{2} & h_{i-2}^{2} \\
h_{i+2}^{3} & h_{i+1}^{3} & -h_{i-1}^{3} & -h_{i-2}^{3} \\
h_{i+2}^{4} & h_{i+1}^{4} & h_{i-1}^{4} & h_{i-2}^{4}
\end{array}\right)}_{\left[\boldsymbol{A}_{4}\right]} \cdot \underbrace{\left(\begin{array}{c}
a_{i+2} \\
a_{i+1} \\
a_{i-1} \\
a_{i-2}
\end{array}\right)}_{\boldsymbol{a}}=\underbrace{\left(\begin{array}{l}
1 \\
0 \\
0 \\
0
\end{array}\right)}_{\boldsymbol{b}},
$$

we must add the equation for $a_{i}=-\left(a_{i-2}+a_{i-2}+a_{i+1}+a_{i+2}\right)$, as it is given by the second of Eq. (A5). Applying Eq. (A6), one finds

$$
\begin{aligned}
\operatorname{det}\left[\boldsymbol{A}_{4}\right]= & h_{i+2} h_{i+1} h_{i-1} h_{i-2}\left(h_{i+2}+h_{i-2}\right)\left(h_{i+1}+h_{i-2}\right) \\
& \times\left(h_{i-1}-h_{i-2}\right)\left(h_{i+2}+h_{i-1}\right)\left(h_{i+1}+h_{i-1}\right)\left(h_{i+1}-h_{i+2}\right) .
\end{aligned}
$$

Finally, using (A6) and (A12) in the first of (A5), we explicitly obtain the sought coefficients as follows: 


$$
\begin{aligned}
& a_{i+2}=\frac{h_{i+1} h_{i-1} h_{i-2}}{h_{i+2}\left(h_{i+2}+h_{i-2}\right)\left(h_{i+2}+h_{i-1}\right)\left(h_{i+1}-h_{i+2}\right)}, \\
& a_{i+1}=\frac{-h_{i+2} h_{i-1} h_{i-2}}{h_{i+1}\left(h_{i+1}+h_{i-2}\right)\left(h_{i+1}+h_{i-1}\right)\left(h_{i+1}-h_{i+2}\right)}, \\
& a_{i-1}=\frac{h_{i+2} h_{i+1} h_{i-2}}{h_{i-1}\left(h_{i+2}+h_{i-1}\right)\left(h_{i+1}+h_{i-1}\right)\left(h_{i-1}-h_{i-2}\right)}, \\
& a_{i-2}=\frac{-h_{i+2} h_{i+1} h_{i-1}}{h_{i-2}\left(h_{i+2}+h_{i-2}\right)\left(h_{i+1}+h_{i-2}\right)\left(h_{i-1}-h_{i-2}\right)} .
\end{aligned}
$$

\section{APPENDIX B: NON-UNIFORM GRID AND MACHINE PRECISION}

The non-uniform grid with a fine spacing around $x=0$ is generated by applying the function

$$
x_{i}=\frac{x_{i}^{*}\left[\left(\frac{2\left|x_{i}^{*}\right|}{L_{x}}\right)^{\beta}+b\right]}{1+b}
$$

to a uniform grid of spacing $h^{*}$ centered around $x_{0}^{*}=0$ and defined by $x_{i}^{*}=i h^{*}$, where $i=-N_{x} / 2, \ldots, N_{x} / 2$, with $N_{x}$ the number of sub-intervals in the interval $\left[-L_{x} / 2, L_{x} / 2\right]$. The choice of the real parameters $\beta>0$ and $b>0$ allows us to control the degree of non-uniformity, which increases as $\beta$ increases and/or $b$ decreases. The generic spacing $h_{i}$ is indeed given in terms of the uniform mesh size $L_{x} / N_{x}$ by the relation

$$
h_{i}=\frac{L_{x}}{N_{x}} \frac{\left[\left(2|i+1| N_{x}\right)^{\beta}+b\right]+i\left(2 N_{x}\right)^{\beta}\left(|i+1|^{\beta}-|i|^{\beta}\right)}{1+b} .
$$

Notice that small values of $h_{i}$ can lead to truncation errors comparable to or smaller than the machine epsilon for a given precision. Therefore, in order to avoid ill-conditioning problems related to the subtraction of too close numbers, both the parameters $\beta$ and $b$ for a fixed machine precision $\varepsilon_{p}$ and the machine precision itself must be made to vary at the varying non-ideal parameters allowing reconnection once the number of grid points $N_{x}+1$ is fixed: on the one hand, the smallest $h_{j}$ must be small enough to allow a sufficient resolution of the reconnecting layer width $\delta$; on the other hand, $h_{j} / a$ must be larger than the machine epsilon.

Lower constraints on $h_{j} / a$ are indeed posed by rounding errors of the order of the machine precision: by taking into account the implicit rounding error of truncated numbers given by $\varepsilon_{p r}$ (e.g., in decimal single precision $p r \rightarrow s, \varepsilon_{s}=5 \times 10^{-7}$ ), Eq. (A1) for $n=2$ and $N_{t}=9$ should be rewritten as

$$
\left.\frac{\partial^{2} f}{\partial x^{2}}\right|_{i}=\sum_{m=-4}^{m=4} a_{i+m}\left(f_{i+m}+\varepsilon_{p r}\right)+e^{7^{t h}} .
$$

It follows that the condition

$$
\left|\frac{\partial^{2} f}{\partial x^{2}}\right|_{x_{i}}-\sum_{m=-4}^{m=4} a_{i+m} f_{m} \mid \leq \underbrace{e_{i}^{\left(7^{\text {th }}\right)}+\sum_{m=-4}^{m=4} a_{i+m} \varepsilon_{p r}}_{\Lambda_{i}\left(h_{i-4}, \ldots, h_{i+4}\right)}
$$

must be respected, where $\Lambda_{i}\left(h_{i-4}, \ldots, h_{i+4}\right)$ is a polynomial function of the mesh spacings $h_{i-4}, \ldots, h_{i+4}$ [cf. Eqs. (A7) and (A13)-(A16)].

In order to find a quantitative threshold on $h_{j}$, we could underestimate $\Lambda_{i}\left(h_{i-4}, \ldots, h_{i+4}\right)$ by means of the function $\Lambda_{i}^{*}\left(\min _{m}\left\{h_{i+m}\right\}\right)$ obtained by calculating $\Lambda_{i}\left(h_{i-4}, \ldots, h_{i+4}\right)$ after substituting, at the varying of $m$, each $h_{i+m}$ with $\min _{m}\left\{h_{i+m}\right\}=h^{*}$. The numerical value of the latter can be, thus, computed by finding the minimum of $\Lambda_{i}^{*}\left(h^{*}\right)$ imposing

$$
\frac{\partial \Lambda_{i}^{*}\left(h^{*}\right)}{\partial h^{*}}=0 .
$$

This gives us a restrictive lower threshold on the smallest value of $h_{j}$ on the grid, which is compatible with a given machine epsilon $\varepsilon_{p}$. If this is not the case, the machine precision must be increased (i.e., $\varepsilon_{p}$ must be decreased).

In the present version of the code, however, we have proceeded in a somehow more "empirical" way, although not optimized, thanks to previous knowledge of relevant parameters in similar non-uniform grid numerical solutions of the eigenvalue problem: first, a set of parameters has been fixed and has been checked to grant convergence of the estimation of $\delta$ for a certain machine epsilon and for some relatively large values of the non-ideal parameters. For example: $N_{x}=1400, L_{x}=4 \pi \quad \beta=4$, and $b=10^{-6}$ for $\varepsilon_{p r}$ $\simeq 10^{-36}$ (quadruple precision) and $S^{-1}=10^{-6}, \rho_{s} / a=5 \times 10^{-2}$, in the warm-resistive case. Then, when the non-ideal parameters are decreased, an outer loop makes $\beta$ and $b$ change in order to refine the non-uniform grid, while an inner loop checks the convergence by numerical calculation of a fourth order derivative (approximated with a fourth order accurate stencil) of a test-function for which an exact analytical solution is known. If convergence is not granted with respect to a certain threshold error that we have chosen, the machine epsilon is decreased for that set of $\beta$ and $b$ until convergence is measured.

\section{DATA AVAILABILITY}

The data that support the findings of this study are available within the article.

\section{REFERENCES}

${ }^{1}$ K. Takeda, O. Agullo, S. Benkadda, A. Sen, N. Bian, and X. Garbet, "Nonlinear viscoresistive dynamics of the $\mathrm{m}=1$ tearing instability," Phys. Plasmas 15, 022502 (2008).

${ }^{2}$ A. Tenerani, A. F. Rappazzo, M. Velli, and F. Pucci, "The tearing mode instability of thin current sheets: The transition to fast reconnection in presence of viscosity," Astrophys. J. 801, 145 (2015).

${ }^{3}$ H. R. Strauss, "Nonlinear three-dimensional magnetohydrodynamics of noncircular tokamaks," Phys. Rev. 19, 134 (1976).

${ }^{4}$ H. R. Strauss, "Dynamics of high- $\beta$ tokamaks," Phys. Rev. 20, 1354 (1977).

${ }^{5}$ D. Grasso, F. Pegoraro, F. Porcelli, and F. Califano, "Hamiltonian magnetic reconnection,” Plasma Phys. Controlled Fusion 41, 1497-1515 (1999).

${ }^{6}$ T. J. Schep, F. Pegoraro, and B. N. Kuvshinov, "Generalized two-fluid theory of nonlinear magnetic structures,” Phys. Plasmas 1, 2843-2852 (1994).

${ }^{7}$ D. Del Sarto, F. Califano, and F. Pegoraro, "Electron parallel compressibility in the nonlinear development of two-dimensional magnetohydrodynamic reconnection," Mod. Phys. Lett. B 20, 931-961 (2006).

${ }^{8} \mathrm{X}$. Wang and A. Batthacharjee, "Nonlinear dynamics of the $\mathrm{m}=1$ instability and fast sawtooth collapse in high temperature plasmas," Phys. Rev. Lett. 70, 1627 (1993). 
${ }^{9}$ R. G. Kleva, J. F. Drake, and F. L. Waelbroeck, "Fast reconnection in high temperature plasmas," Phys. Plasmas 2, 23 (1995).

${ }^{10}$ R. Fitzpatrick and F. Porcelli, "Collisionless magnetic reconnection with arbitrary guide field," Phys. Plasmas 11, 4713 (2004).

${ }^{11} \mathrm{~N}$. H. Bian and D. Tsiklauri, "Compressible hall magnetohydrodynamics in a strong magnetic field," Phys. Plasmas 16, 064503 (2009).

${ }^{12}$ A. Hasegawa and C. Uberoi, "The Alfvén Wave," Report No. DOE/TIC-11197, Technical Information Center, United State Department of Energy, Springfield, VA, 1982.

${ }^{13}$ H. P. Furth, J. Killeen, and M. N. Rosenbluth, "Finite-resistivity instabilities of a sheet pinch,” Phys. Fluids 6, 459-484 (1963).

${ }^{14}$ B. Coppi, R. Galvao, R. Pellat, M. Rosenbluth, and P. Rutherford, "Resistive internal kink modes," Soviet Journal of Plasma Physics 2, 961-966 (1976).

${ }^{15}$ G. Ara, B. Basu, B. Coppi, G. Laval, M. N. Rosenbluth, and B. V. Waddell, "Magnetic reconnection and $\mathrm{m}=1$ oscillations in current carrying plasmas," Ann. Phys. 112, 443-476 (1978).

${ }^{16}$ B. Coppi, “Inertial' instabilities in plasmas," Phys. Lett. 11, 226-228 (1964).

${ }^{17} \mathrm{~F}$. Pegoraro, F. Porcelli, and T. J. Schep, "Internal kink modes in the ion-kinetic regime," Phys. Fluids B 1, 364-374 (1989).

${ }^{18} \mathrm{~F}$. Pegoraro and T. J. Schep, "Theory of resistive modes in the ballooning representation," Plasma Phys. Controlled Fusion 28, 647-667 (1986).

${ }^{19}$ J. F. Drake and Y. C. Lee, "Kinetic theory of tearing instabilities," Phys. Fluids B 20, 1341 (1977).

${ }^{20} \mathrm{P}$. Holoborodko, "Advanpix multiprecision computing tool for MATLAB," https://www.advanpix.com (2012).

${ }^{21}$ G. H. Golub and C. F. Van Loan, Matrix Computations (John Hopkins University Press, 1996).

${ }^{22}$ F. Porcelli, "Viscous resistive magnetic reconnection," Phys. Fluids 30, 1734-1742 (1987).

${ }^{23} \mathrm{~A}$. Zocco and A. A. Schekochihin, "Fast linear growth of collisionless double tearing modes in a cylindrical plasma," Phys. Plasmas 18, 102309 (2011).

${ }^{24}$ H. P. Furth, P. H. Rutherford, and H. Selberg, "Tearing mode in the cylindrical tokamak," Phys. Fluids 16, 1054 (1973).

${ }^{25}$ F. Porcelli, D. Borgogno, F. Califano, D. Grasso, M. Ottaviani, and F. Pegoraro, "Recent advances in collisionless magnetic reconnection," Plasma Phys. Controlled Fusion 44, B389-B405 (2002).

${ }^{26}$ D. Grasso, R. J. Hastie, F. Porcelli, and C. Tebaldi, "Critical $\Delta^{\prime}$ for stability of viscoresistive tearing modes," Phys. Plasmas 15, 072113 (2008).

${ }^{27}$ F. Porcelli, "Collisionless $m=1$ tearing mode," Phys. Rev. Lett. 66, 425 (1991).

${ }^{28}$ E. G. Harris, "On a plasma sheath separating regions of oppositely directed magnetic field," Il Nuovo Cimento 23, 115-121 (1962).

${ }^{29}$ D. Del Sarto, F. Pucci, A. Tenerani, and M. Velli, "'Ideal' tearing and the transition to fast reconnection in the weakly collisional MHD and EMHD regimes," J. Geophys. Res. 121, 1857-1873, https://doi.org/10.1002/2015JA021975 (2016).

${ }^{30}$ F. Pucci, M. Velli, A. Tenerani, and D. Del Sarto, "Onset of fast "ideal" tearing in thin current sheets: Dependence on the equilibrium current profile," Phys. Plasmas 25, 032113 (2018).

${ }^{31}$ M. A. Cross and G. Van Hoven, "Magnetic and gravitational energy release by resistive instabilities," Phys. Rev. A 4, 2347 (1971).

${ }^{32}$ G. Van Hoven and M. A. Cross, "Resistive instability of a sheet pinch," Phys. Fluids 14, 1141 (1971).

${ }^{33} \mathrm{G}$. Van Hoven and M. A. Cross, "Inertial magnetic field reconnection and magnetospheric substorms," Phys. Rev. Lett. 30, 642 (1973).

${ }^{34}$ G. Van Hoven and M. A. Cross, "Energy release by magnetic tearing: The nonlinear limit," Phys. Rev. A 7, 1347 (1973).

${ }^{35} \mathrm{~J}$. W. Dungey, "Some researches in cosmic magnetism," Ph.D. thesis (Cambridge University, Cambridge, UK, 1950).

${ }^{36} \mathrm{~J}$. W. Dungey, "Conditions for the occurrence of electrical discharges in astrophysical systems," London Edinburgh Dublin Philos. Mag. 29, 752-738 (1953).

${ }^{37}$ B. Coppi, G. Laval, and R. Pellat, "Dynamics of the geomagnetic tail," Phys. Rev. Lett. 16, 1207 (1966).

${ }^{38}$ J. H. Piddington, "A theory of auroras and the ring current," J. Atmos. Sol.Terr. Phys. 29, 87-105 (1967).

${ }^{39} \mathrm{~B}$. Coppi and A. B. Friedland, "Processes of magnetic-energy conversion and solar flares," Astrophys. J. 169, 379 (1971).

${ }^{40}$ E. R. Priest, "Current sheet models of solar flares," Sol. Phys. 47, 41-75 (1976).
${ }^{41} \mathrm{M}$. Velli and A. W. Hood, "Resistive tearing in line-tied magnetic fields: Slab geometry," Sol. Phys. 119, 107-124 (1989).

${ }^{42} \mathrm{~K}$. Shibata and S. Tanuma, "Plasmoid-induced-reconnection and fractal reconnection," Earth Planets Space 53, 473-482 (2001).

${ }^{43}$ R. G. Giovanelli, "A theory of chromospheric flares," Nature 158, 81 (1946).

${ }^{44} \mathrm{~F}$. Hoyle, Some Recent Researches in Solar Physics (Cambrdige University Press, Cambridge, UK, 1949).

${ }^{45}$ J. W. Dungey, Cosmic Electrodynamics (Cambrdige University Press, 1958).

${ }^{46} \mathrm{G}$. Einaudi and F. Rubini, "Resistive instabilities in a flowing plasma. II. Effects of viscosity," Phys. Fluids B 1, 2224 (1989).

${ }^{47} \mathrm{~L}$. Ofman, X. L. Chen, and P. J. Morrison, "Resistive tearing mode instability with shear flow and viscosity," Phys. Plasmas B 3, 1346 (1991).

${ }^{48} \mathrm{~N}$. H. Bian and G. Vekstein, "Energetic of forced magnetic reconnection in a visco-resistive plasma," Phys. Plasmas 12, 072902 (2005).

${ }^{49} \mathrm{~F}$. Pucci and M. Velli, "Reconnection of quasi-singular current sheets: The "ideal" tearing mode," Astrophys. J. Lett. 780, L19 (2014).

${ }^{50}$ L. Comisso, D. Grasso, F. L. Waelbroeck, and D. Borgogno, "Gyro-induced acceleration of magnetic reconnection," Phys. Plasmas 20, 092118 (2013).

${ }^{51}$ F. Pegoraro and T. J. Schep, "Low-frequency modes with high toroidal mode numbers: A general formulation," Phys. Fluids 24, 478 (1981).

${ }^{52}$ W. G. Hammett, W. Dorland, and F. W. Perkins, "Fluid models of phase mixing, Landau damping, and nonlinear gyrokinetic dynamics," Phys. Fluids B $\mathbf{4}$, 2052 (1992).

${ }^{53}$ F. L. Waelbroeck, R. D. Hazeltine, and P. J. Morrison, "A Hamiltonian electromagnetic gyrofluid model," Phys. Plasmas 16, 032109 (2009).

${ }^{54}$ E. Tassi, P. J. Morrison, F. L. Waelbroeck, and D. Grasso, "Hamiltonian formulation and analysis of a collisionless fluid reconnection model," Plasma Phys. Controlled Fusion 50, 085014 (2008).

${ }^{55}$ D. Del Sarto, C. Marchetto, F. Pegoraro, and F. Califano, "Finite Larmor radius effects in the nonlinear dynamics of collisionless magnetic reconnection," Plasma Phys. Controlled Fusion 53, 035008 (2011).

${ }^{56} \mathrm{M}$. Ottaviani and F. Porcelli, "Fast nonlinear magnetic reconnection," Phys. Plasmas 2, 4104 (1995)

${ }^{57}$ F. Pegoraro, D. Borgogno, F. Califano, E. Echkina, D. Grasso, T. Liseikina, and F. Porcelli, "Developments in the theory of collisionless reconnection in configurations with a strong guide field," Nonlinear Proc. Geophys. 11, 567 (2004).

${ }^{58} \mathrm{M}$. Ottaviani and F. Porcelli, "Nonlinear collisionless magnetic reconnection," Phys. Rev. Lett. 71, 3802 (1993).

${ }^{59} \mathrm{D}$. Del Sarto and M. Ottaviani, "Secondary fast reconnecting instability in the sawtooth crash," Phys. Plasmas 24, 012102 (2017).

${ }^{60} \mathrm{P}$. A. Cassak and J. F. Drake, "The impact of microscopic magnetic reconnection on pre-flare energy storage," Astrophys. J. Lett. 707, L158 (2009).

${ }^{61}$ D. Del Sarto, M. Ottaviani, F. Pucci, A. Tenerani, and M. Velli, "Spontaneous magnetic reconnection of thin current sheets," in Comptes-Rendus de la $21^{e}$ Rencontre du Non Linéaire, Paris 2018, edited by É. Falcon, M. Lefranc, F. Pétréluis, and C.-T. Pham (Université Paris Diderot, Non-Linéaire Publications, Paris, France, 2018), Vol. 21, pp. 13-18.

${ }^{62}$ A. Tenerani, M. Velli, A. F. Rappazzo, and F. Pucci, "Magnetic reconnection: Recursive current sheet collapse triggered by 'ideal' tearing," Astrophys. J. Lett. 813, L32 (2015).

${ }^{63}$ T. Tajima and K. Shibata, Plasma Astrophysics (Addison-Wesley, 1997), p. 229.

${ }^{64} \mathrm{~N}$. Loureiro, A. Schekochihin, and S. Cowley, "Instability of current sheets and formation of plasmoid chains," Phys. Plasmas 14, 100703 (2007).

${ }^{65}$ A. Bhattacharjee, Y.-M. Huang, H. Yang, and B. Rogers, "Fast reconnection in high-lundquist-number plasmas due to the plasmoid instability," Phys. Plasmas 16, 112102 (2009).

${ }^{66}$ L. Comisso and D. Grasso, "Visco-resistive plasmoid instability," Phys. Plasmas 23, 032111 (2016).

${ }^{67}$ A. Tenerani, M. Velli, A. F. Rappazzo, and F. Pucci, "Ideally' unstable current sheets and the triggering of fast magnetic reconnection," J. Plasma Phys. 82, 535820501 (2016).

${ }^{68}$ S. K. Lele, "Compact finite difference schemes with spectral-like resolution," J. Comp. Phys. 103, 16-42 (1992).

${ }^{69}$ D. Hoffman, Numerical methods for scientists and engineers (CRC Press Taylor \& Francis Group, Boca Raton, FL, 2001).

${ }^{70}$ G. P. Zank and W. H. Matthaeus, "The equations of reduced magnetohydrodynamics," J. Plasma Phys. 48, 85 (1992). 